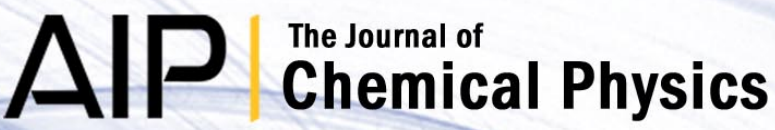

A comparative study of the $\mathrm{Si}+\mathrm{O} 2 \rightarrow \mathrm{SiO}+\mathrm{O}$ reaction dynamics from quasiclassical trajectory and statistical based methods

Fabrice Dayou, Pascal Larrégaray, Laurent Bonnet, Jean-Claude Rayez, Pedro Nilo Arenas et al.

Citation: J. Chem. Phys. 128, 174307 (2008); doi: 10.1063/1.2913156

View online: http://dx.doi.org/10.1063/1.2913156

View Table of Contents: http://jcp.aip.org/resource/1/JCPSA6/v128/i17

Published by the AIP Publishing LLC.

Additional information on J. Chem. Phys.

Journal Homepage: http://jcp.aip.org/

Journal Information: http://jcp.aip.org/about/about_the_journal

Top downloads: http://jcp.aip.org/features/most_downloaded

Information for Authors: http://jcp.aip.org/authors

\section{ADVERTISEMENT}

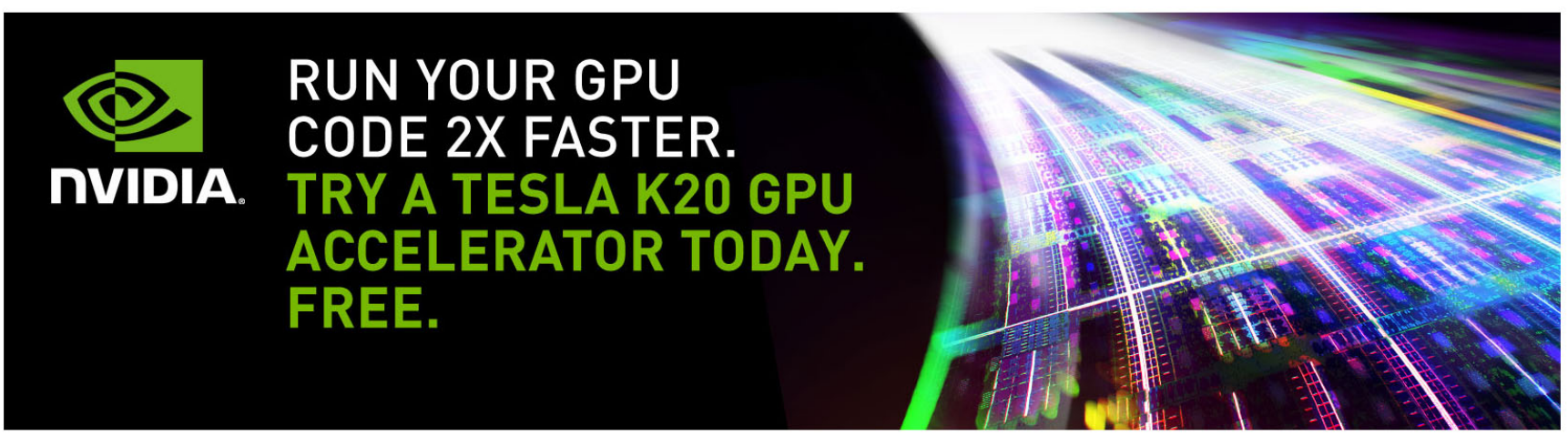




\title{
A comparative study of the $\mathrm{Si}+\mathrm{O}_{2} \rightarrow \mathrm{SiO}+\mathrm{O}$ reaction dynamics from quasiclassical trajectory and statistical based methods
}

\author{
Fabrice Dayou, ${ }^{1, a)}$ Pascal Larrégaray, ${ }^{2}$ Laurent Bonnet, ${ }^{2}$ Jean-Claude Rayez, ${ }^{2}$ \\ Pedro Nilo Arenas, ${ }^{3}$ and Tomás González-Lezana ${ }^{3}$ \\ ${ }^{1}$ Laboratoire d'Etude du Rayonnement et de la Matière en Astrophysique, UMR 8112 du CNRS, \\ Observatoire de Paris-Meudon, 5 Place Jules Janssen, 92195 Meudon Cedex, France \\ ${ }^{2}$ Institut des Sciences Moléculaires, UMR 5255 du CNRS, Université Bordeaux 1, \\ 351 Cours de la libération, 33405 Talence Cedex, France \\ ${ }^{3}$ Instituto de Física Fundamental, Consejo Superior de Investigaciones Científicas, \\ Serrano 123, 28006 Madrid, Spain
}

(Received 22 February 2008; accepted 1 April 2008; published online 7 May 2008)

\begin{abstract}
The dynamics of the singlet channel of the $\mathrm{Si}+\mathrm{O}_{2} \rightarrow \mathrm{SiO}+\mathrm{O}$ reaction is investigated by means of quasiclassical trajectory (QCT) calculations and two statistical based methods, the statistical quantum method (SQM) and a semiclassical version of phase space theory (PST). The dynamics calculations have been performed on the ground ${ }^{1} A^{\prime}$ potential energy surface of Dayou and Spielfiedel [J. Chem. Phys. 119, $4237(2003)]$ for a wide range of collision energies $\left(E_{c}\right.$ $=5-400 \mathrm{meV})$ and initial $\mathrm{O}_{2}$ rotational states $(j=1-13)$. The overall dynamics is found to be highly sensitive to the selected initial conditions of the reaction, the increase in either the collisional energy or the $\mathrm{O}_{2}$ rotational excitation giving rise to a continuous transition from a direct abstraction mechanism to an indirect insertion mechanism. The product state properties associated with a given collision energy of $135 \mathrm{meV}$ and low rotational excitation of $\mathrm{O}_{2}$ are found to be consistent with the inverted $\mathrm{SiO}$ vibrational state distribution observed in a recent experiment. The SQM and PST statistical approaches, especially designed to deal with complex-forming reactions, provide an accurate description of the QCT total integral cross sections and opacity functions for all cases studied. The ability of such statistical treatments in providing reliable product state properties for a reaction dominated by a competition between abstraction and insertion pathways is carefully examined, and it is shown that a valuable information can be extracted over a wide range of selected initial conditions. () 2008 American Institute of Physics. [DOI: 10.1063/1.2913156]
\end{abstract}

\section{INTRODUCTION}

The title reaction has been the subject of various experimental and theoretical investigations in the last five years. The $\mathrm{Si}\left({ }^{3} P\right)+\mathrm{O}_{2}\left(X^{3} \Sigma_{g}^{-}\right)$reaction kinetics has been studied from room to low temperatures, where it can exothermically proceed through the ground singlet ${ }^{1} A^{\prime}$ and first triplet ${ }^{3} A^{\prime}$ electronic states, ${ }^{1}$ leading to the $\mathrm{SiO}\left(X^{1} \Sigma^{+}\right)+\mathrm{O}\left({ }^{1} D\right)$ and $\mathrm{SiO}\left(X^{1} \Sigma^{+}\right)+\mathrm{O}\left({ }^{3} P\right)$ products, respectively. This reaction is usually invoked as one of the main source of $\mathrm{SiO}$ in the interstellar medium (ISM), and the aim was to inquire if the temperature dependence of the reaction kinetics is at the origin of the huge variation of abundance of $\mathrm{SiO}$ recorded in the ISM under different physical conditions. The thermal rate constant has been experimentally measured ${ }^{2}$ in the range of $15-300 \mathrm{~K}$, pointing out to a high reactivity for the whole range of temperatures. This result was further confirmed by theory, although the adiabatic capture centrifugal sudden (CS) approximation ${ }^{3}$ and quasiclassical trajectory ${ }^{1}$ (QCT) approaches yielded rate constant values somewhat lower than experiment. Such a discrepancy was attributed to the role of the first triplet ${ }^{3} A^{\prime}$ state, whose contribution to reactivity was introduced in an approximate manner in both the-

\footnotetext{
${ }^{a)}$ Author to whom correspondence should be adressed. Electronic mail: fabrice.dayou@obspm.fr.
}

oretical calculations. Further evidences of the relevance of both singlet and triplet channels in the title reaction can be retrieved from recent $a b$ initio quantum chemistry calculations of the minimum energy paths of distinct spin multiplicity associated with the dissociation of the $\mathrm{SiO}_{2}$ molecule., The primary goal of such studies was to investigate the structure and energetic properties of small $\mathrm{Si}-\mathrm{O}$ clusters, species highly relevant to the field of silicon-based microelectronic devices (see, e.g., Ref. 6 and references therein), and they indicate the absence of any barrier to reactivity for both singlet and triplet channels. More recently, rovibrational distributions of the product $\mathrm{SiO}\left(X^{1} \Sigma^{+}\right)$molecules and the relative populations of the spin-orbit fine-structure states of $\mathrm{O}\left({ }^{3} P_{J}\right)$ could be measured at a given collision energy by Yamashiro et al. by using a crossed-beam laser-induced fluorescence technique. ${ }^{7}$ The results show a nonstatistical population of the $\mathrm{O}\left({ }^{3} P_{J}\right)$ fine-structure levels, as well as an inverted vibrational distribution of the $\mathrm{SiO}\left(\nu^{\prime}\right)$ molecules, with peaks at $\nu^{\prime}=0,4$, and 7-8. Such a feature suggests the existence of distinct reaction mechanisms, possibly related to the specific contribution to reactivity of the singlet and triplet channels at the collision energy considered in experiment $(135 \mathrm{meV})$.

The conclusions drawn by a recent QCT study ${ }^{8}$ of the singlet channel of the reaction support-at least partiallythese experimental findings. The collision dynamics rested 
on the ${ }^{1} A^{\prime}$ potential energy surface (PES) of Ref. 1, which is barrierless both in the entrance and exit channels (with an exoergicity of about $1 \mathrm{eV}$ ), and displayed three deep minima corresponding to the linear $\mathrm{SiOO}\left({ }^{1} \Sigma^{+}\right)$, triangular $\mathrm{OSiO}$ $\left({ }^{1} A_{1}\right)$, and linear OSiO $\left({ }^{1} \Sigma_{g}^{+}\right)$species (hereafter referred as MIN1, MIN2, and MIN3, respectively). Such a picture of the singlet channel is in close agreement with the ab initio results of Refs. 4 and 5, although the characterization of the linear species MIN1 had not been undertaken in these studies. At low collision energies (typically below $200 \mathrm{meV}$ ), the reaction was found to yield product $\mathrm{SiO}$ molecules characterized by an inverted vibrational distribution peaked at $\nu^{\prime}=3$, low rotational excitation, and preferential backward scattering. These features clearly depart from the statistical energy disposal into the product modes that one would expect from the presence of quite deep potential wells along the reaction path $(-2.7,-4.8$, and $-7.1 \mathrm{eV}$ for MIN1, MIN2, and MIN3, respectively). In fact, in this low collision energy regime, the reaction was found to proceed through a direct abstraction mechanism involving a short-lived intermediate complex, the linear species MIN1. On the contrary, at higher collision energies, the product state distributions are fairly well described by prior distributions, ${ }^{9}$ indicating a near statistical energy disposal and random angular scattering. This originates from a dominant contribution of an insertion mechanism involving two long-lived intermediates, the bent and linear OSiO species, MIN2 and MIN3.

The singlet channel of the $\mathrm{Si}+\mathrm{O}_{2}$ reaction can, thus, be seen as a prototypical system for which the reaction mechanisms smoothly evolve from abstraction to insertion on increasing the collision energy, giving rise to well-marked differences in the features of the products. The existence of extremely deep potential wells in the PES, and the long lifetime of the associated intermediate species, renders the application of exact quantum mechanical (EQM) methods to treat the dynamics (a first attempt ${ }^{10}$ by means of a wave packet method showed that the collision lifetime is on the picosecond scale) very challenging. It is, thus, worthwhile to test the ability of statistical based methods-designed to deal with complex-forming reactions-to provide a reliable description of the reaction attributes. This is the purpose of the present work, where we examine in further details the dynamics of the $\mathrm{Si}+\mathrm{O}_{2}$ reaction through a confrontation between the QCT approach and extended statistical treatments. To this end, we employ the recently developed statistical quantum method ${ }^{11,12}$ (SQM) and a semiclassical version of phase space theory ${ }^{13}$ (PST). The underlying assumption to such treatments is that the reaction proceeds by formation of an intermediate complex, with a lifetime sufficiently long to completely randomize the energy partitioning into the product modes. In such a case, the formation and subsequent fragmentation of the intermediate complex can be separately treated by means of capture probabilities. It is worth noticing that the present statistical treatments account for the conservation requirements on the total energy, parity, and total angular momentum values and, in the particular case of the SQM method, rely on an accurate description of long-range inelastic couplings in both the reactant and product arrangements. The SQM and PST approaches, thus, allow us to in- corporate potentially important effects which were neglected in the previous statistical treatment ${ }^{8}$ of the $\mathrm{Si}+\mathrm{O}_{2}$ reaction, where the conservation of total energy was the unique requirement to prior distributions.

The reaction attributes considered in the present work are the total integral cross sections (ICSs), opacity functions, product state distributions, and differential cross sections (DCSs). The QCT and SQM approaches employed here are quite similar to that previously reported, ${ }^{8,12}$ whereas significant modifications to the PST approach used in Ref. 13 had been necessary to account for the nonzero rotational angular momentum of the $\mathrm{O}_{2}$ molecule. A semiclassical parity restoring correction to the usual PST method is also applied in this work. Indeed, it has been recently shown ${ }^{14,15}$ that the inability of classical approaches to properly reproduce the sharp forward/backward peaks in the DCSs relies on the neglect of the parity conservation requirement. These findings have been successfully applied to the $\mathrm{H}^{+}+\mathrm{H}_{2}$ and $\mathrm{H}^{+}+\mathrm{D}_{2}$ reactions, where a QCT version of the SQM approach in which parity conservation is explicitly included ${ }^{16}$ allowed us to get DCSs exhibiting the right behavior, in clear contrast to the QCT results ${ }^{17}$ previously reported.

Along the present study of the $\mathrm{Si}+\mathrm{O}_{2}$ reaction dynamics, a particular emphasis will be given on the dependence of the reaction mechanisms on collision energy and, as a new issue with respect to previous works, ${ }^{8}$ on their dependence on the selected rotational state of the $\mathrm{O}_{2}$ molecule. It is worth mentioning that the statistical treatments employed here are generally applied to complex-forming reactions characterized by a dominant insertion mechanism. The close agreement between experimental measurements and EQM, SQM, and PST calculations for a variety of $\mathrm{X}+\mathrm{H}_{2} \rightarrow \mathrm{XH}+\mathrm{H}$ insertion reactions ${ }^{12,13,17-20}\left[\right.$ with $\mathrm{X}=\mathrm{C}\left({ }^{1} D\right) \mathrm{S}\left({ }^{1} D\right), \mathrm{O}\left({ }^{1} D\right), \mathrm{N}\left({ }^{1} D\right)$, and $\left.\mathrm{H}^{+}\right]$demonstrates the validity of statistical approaches in such a case (nonadiabatic effects are usually invoked to explain significant discrepancies, such as in the $\mathrm{O}+\mathrm{O}_{2}$ insertion reaction ${ }^{21,22}$ ). The $\mathrm{Si}+\mathrm{O}_{2}$ reaction under study is, however, characterized by a competition between abstraction and insertion mechanisms and, by definition, the statistical approaches are not expected to yield a proper description of the dynamical features due to abstraction. Instead, they will serve to determine how statistical is the reaction under given initial conditions and to discriminate the sensitivity of each reaction attribute to the microscopic mechanisms at works. In this sense, the present study is closely related to that undertaken on the $\mathrm{H}+\mathrm{O}_{2}$ endothermic reaction-by means of SQM and PST approaches and time-dependent quantum calculations $^{23}$-for which a transition from an insertion mechanism to a direct abstraction pathway had been identified $^{24,25}$ on increasing collision energy. A noteworthy difference from the present case is that the insertion-toabstraction transition is followed by an increasing number of inelastic events, driven by the strong interaction region of the PES, which cannot be properly accounted for by statistical approaches. This is in clear contrast to the features of the $\mathrm{Si}+\mathrm{O}_{2}$ reaction, for which the complex formation is almost entirely followed by reactive events, even in the case of the direct abstraction mechanism. The title reaction, hence, offers a good opportunity to investigate the ability of statistical 
treatments to provide a reliable description of a given class of reaction attributes (opacity functions and ICS) in the case of a dominant abstraction mechanism.

\section{THEORY}

\section{A. Quasiclassical trajectory calculations}

The QCT calculations have been performed by running batches of $5 \times 10^{4}$ trajectories at nine collision energies $(5,10,25,50,100,200,300,400$, and $600 \mathrm{meV})$ for each of the first eight rotational levels $j=1-15$ of $\mathrm{O}_{2}(\nu=0)$. The calculations have been carried out by means of a QCT code developed by Halvick and Rayez, ${ }^{26}$ already employed in previous studies of the $\mathrm{Si}+\mathrm{O}_{2}$ reaction. ${ }^{1,8}$ The procedure is based on a standard Monte Carlo sampling of the initial conditions, ${ }^{27}$ where an initial separation of $18 a_{0}$ between the reactants is chosen. The Burlisch-Stoer method is used to integrate the set of Hamilton equations, with an integration time step of $5 \times 10^{-16} \mathrm{~s}$. This was found to be sufficient to ensure a conservation of total energy and total angular momentum of $10^{-9}-10^{-10}$ unity. The large reaction probability provides statistical deviations lower than $0.5 \%$ on the resulting cross section values. The assignment of the product quantum numbers $\left(\nu^{\prime}, j^{\prime}\right)$ is performed by equating the classical rotational angular momentum to $\hbar\left[j^{\prime}\left(j^{\prime}+1\right)\right]^{1 / 2}$, followed by an iterative procedure, where a Dunham expansion $\left(\omega_{e}, \omega_{e} x_{e}, B_{e}, \alpha_{e}\right.$ and $\left.D_{e}\right)$ adjusted to the diatomic part of the PES is employed to express the internal energy of the molecule. The resulting $\left(\nu^{\prime}, j^{\prime}\right)$ values are finally rounded to their nearest integers according to the usual histogram binning method. The known drawbacks of the latter method ${ }^{28,29}$ are expected to have a small influence on the calculated product properties in the present case, owing to the rather small vibrational and rotational spacings of the $\mathrm{SiO}$ molecule together with the exoergic character of the reaction. It is worth mentioning that the total angular momentum values $J$ are selected for each trajectory according to the impact parameter $b$ values, continuously sampled in a $b d b$ distribution. The latter procedure is $\mathrm{known}^{30}$ to introduce a bias toward high $J$ values in the sampling of initial conditions, hence, leading to a higher statistical uncertainty on the reaction probabilities $P_{r}(J)$ corresponding to low $J$ values. The large number of reactive events sampled within the total number of trajectories tends to reduce somewhat such a bias. Nonetheless, the huge number of orbital angular momentum values contributing to reactive events at high collision energies led us to increase the size of the bins to two unities to store the $P_{r}(J)$ values.

\section{B. Statistical quantum method}

Since its initial derivation by Manolopoulos and co-workers, ${ }^{11,22}$ the SQM has been extensively employed to treat a variety of complex-forming atom-diatom reactions. The basic assumptions to ensure the validity of the model is that all reactive events proceed by formation of an intermediate complex and that the lifetime of this complex is long enough to enable a separate treatment of its formation and subsequent fragmentation. According to this model, the stateto-state reaction probability for a total angular momentum $J$ and a parity eigenvalue $I$ is approximated as

$$
\left|S_{\alpha^{\prime} \nu^{\prime} j^{\prime} \Omega^{\prime}, \alpha \nu j \Omega}^{I J}(E)\right|^{2} \simeq \frac{p_{\nu^{\prime} j^{\prime} \Omega^{\prime}}^{I J \alpha^{\prime}}(E) p_{\nu j \Omega}^{I J \alpha}(E)}{\sum_{\alpha^{\prime \prime} \nu^{\prime \prime} j^{\prime \prime} \Omega^{\prime \prime}} p_{\nu^{\prime \prime} j^{\prime \prime} \Omega^{\prime \prime}}^{I J \alpha^{\prime \prime}}(E)},
$$

where $p_{\nu j \Omega}^{I J \alpha}$ and $p_{\nu^{\prime} j^{\prime} \Omega^{\prime}}^{I J \alpha^{\prime}}$ are the capture probabilities for the $\nu j \Omega$ reactant and $\nu^{\prime} j^{\prime} \Omega^{\prime}$ product channels, respectively. The parity-adapted helicity representation is employed in Eq. (1), $\Omega$ being the modulus of the projection of the diatomic rotational angular momentum on the atom-diatom axis.

The capture probabilities are separately calculated in each arrangement $\alpha$ as

$$
p_{\nu j \Omega}^{I J \alpha}(E)=1-\sum_{\nu^{\prime} j^{\prime} \Omega^{\prime}}\left|S_{\nu^{\prime} j^{\prime} \Omega^{\prime}, \nu j \Omega}^{I J \alpha}(E)\right|^{2},
$$

where the sum runs over all the energetically open channels within the arrangement considered. The scattering matrix of Eq. (2) is obtained for each chemical arrangement by solving within the CS approximation ${ }^{31-33}$ the corresponding closecoupled equations. ${ }^{1}$ Those equations are solved by means of a $\log$ derivative propagation ${ }^{34}$ between the asymptotic region and a specific capture radius, which defines the region where the intermediate collision complex is assumed to be formed. In the present work, such integration has been performed from an atom-diatom separation of 4.7 up to $16.5 a_{0}$ for the $\mathrm{Si}+\mathrm{O}_{2}$ arrangement and from 6 to $15 a_{0}$ for the $\mathrm{SiO}+\mathrm{O}$ arrangement. The calculations have been carried out in such a way to compute the reaction attributes for the $5-400 \mathrm{meV}$ collision energy range and the $j=1-13$ rotational levels of $\mathrm{O}_{2}(v=0)$. It is worth mentioning that, even within the CS approximation of the SQM approach, the huge number of total angular momentum values contributing to reactivity renders the quantum dynamical treatment of the reaction quite costly for kinetic energies higher than $400 \mathrm{meV}$.

The statistical state-to-state ICS is calculated from the EQM expression,

$$
\begin{aligned}
\sigma_{\alpha^{\prime} \nu^{\prime} j^{\prime}, \alpha \nu j}(E)= & \frac{\pi}{k_{\alpha v j}^{2}(2 j+1)} \sum_{I J \Omega \Omega^{\prime}}(2 J+1) \\
& \times\left|S_{\alpha^{\prime} \nu^{\prime} j^{\prime} \Omega^{\prime}, \alpha v j \Omega}^{I J}(E)\right|^{2},
\end{aligned}
$$

where $k_{\alpha v j}^{2}=2 \mu\left(E-E_{v j}\right) / \hbar^{2}, E_{v j}$ being the energy of the initial rovibrational state $v j$ of the reactant diatom, and the state-to-state probability $\left|S_{\alpha^{\prime} v^{\prime} j^{\prime} \Omega^{\prime}, \alpha v j \Omega}^{I J}(E)\right|^{2}$ is taken from Eq. (1). Since the SQM approach cannot provide any information regarding the phase of the scattering matrix, the calculation of DCSs requires the use of a random phase approximation. ${ }^{12}$ Within this approximation, the statistical state-to-state DCS is given by

$$
\begin{aligned}
\sigma_{\alpha^{\prime} \nu^{\prime} j^{\prime}, \alpha v j}(\theta, E) \simeq & \frac{1}{8 k_{\alpha \nu j}^{2}} \frac{1}{2 j+1} \sum_{I J \Omega^{\prime} \Omega}(2 J+1)^{2} \\
& \times\left[d_{\Omega^{\prime} \Omega}^{J}(\pi-\theta)^{2}+d_{\Omega^{\prime} \Omega}^{J}(\theta)^{2}\right] \\
& \times\left|S_{\alpha^{\prime} \nu^{\prime} j^{\prime} \Omega^{\prime}, \alpha v j \Omega}^{I J}(E)\right|^{2},
\end{aligned}
$$


where $\theta$ is the center-of-mass (c.m.) scattering angle and $d_{\Omega^{\prime} \Omega}^{J}(\theta)$ the reduced rotation matrix elements. ${ }^{35}$

\section{Phase space theory}

The PST, ${ }^{36-40}$ initially developed to describe the dynamics of complex-forming reactions governed by long-range isotropic forces, has been extensively used during the last 40 years. ${ }^{41}$ The underlying assumption to PST is-like in the SQM case - that all reactive events proceed through a longlived intermediate complex. Within the PST, the probability of formation of the collision complex is estimated via a classical capture model, ${ }^{42}$ whereas the decay probability from the complex to any given product is supposed to be proportional to the ratio of the phase space available to that product, divided by the total phase space consistent with conservation of total energy and total angular momentum values. Within the framework of atom-diatom insertion reactions, a recently developed semiclassical version of PST (Ref. 13) was found to fairly reproduce the ICSs and DCSs provided by EQM and SQM calculations. However, this study was restricted to processes involving a rotationally frozen reactant diatom $(j=0)$. To account for the specificity of the $\mathrm{O}_{2}\left(X^{3} \Sigma_{g}^{-}\right)$molecule (only odd rotational quantum numbers are allowed due to nuclear spin statistics), the PST approach employed in the present work extends the previous one to the general $(j \neq 0)$ case. Within this PST approach, the state-tostate DCS may be written as

$$
\sigma_{\nu^{\prime} j^{\prime}, \nu j}\left(\theta, E_{c}\right)=\frac{1}{k^{2} \sin \theta} \int_{0}^{l_{\max }} P_{\nu^{\prime} j^{\prime}, \nu j}\left(\theta, l, E_{c}\right) l d l,
$$

where $k$ is the reactant momentum and $P_{v^{\prime} j^{\prime}, v j}\left(\theta, l, E_{c}\right)$ is the probability to form the products in the $\left(v^{\prime}, j^{\prime}\right)$ state at the c.m. scattering angle $\theta$ from the initial $(v, j)$ state, consistent with the orbital angular momentum $l$ and collision energy $E_{c}$ values.

Although PST leads to results in fair agreement with more refined quantum treatments, such an approach is unable to describe the sharp forward/backward polarization peaks appearing in the DCSs computed by EQM or SQM calculations. $^{43}$ Some of us recently showed that the fundamental reason for this discrepancy is the neglect of parity properties in classical mechanics. A semiclassical parity restoring correction has, thus, been proposed to circumvent this shortcoming within the framework of complex-forming chemical processes. ${ }^{14,44}$ By following this correction, the parity-corrected DCS $\sigma_{v^{\prime} j^{\prime}, v j}^{\mathrm{pc}}\left(\theta, E_{c}\right)$ may be computed as

$$
\begin{aligned}
\sigma_{\nu^{\prime} j^{\prime}, \nu j}^{\mathrm{pc}}\left(\theta, E_{c}\right)= & \sigma_{\nu^{\prime} j^{\prime}, \nu j}\left(E_{c}\right) \\
& \times \frac{\sigma_{\nu^{\prime} j^{\prime}\left(\Omega^{\prime}=0\right), \nu j(\Omega=0)}\left(\theta, E_{c}\right)+\sigma_{\nu^{\prime} j^{\prime}, \nu j}\left(\theta, E_{c}\right)}{\sigma_{\nu^{\prime} j^{\prime}\left(\Omega^{\prime}=0\right), \nu j(\Omega=0)}\left(E_{c}\right)+\sigma_{\nu^{\prime} j^{\prime}, \nu j}\left(E_{c}\right)},
\end{aligned}
$$

where $\sigma_{v^{\prime} j^{\prime}\left(\Omega^{\prime}=0\right), v j(\Omega=0)}\left(\theta, E_{c}\right)$ is the helicity-resolved DCS for collisions connecting zero-helicity reactants to zerohelicity products $\left(\Omega=\Omega^{\prime}=0\right)$, and $\sigma_{v^{\prime} j^{\prime}\left(\Omega^{\prime}=0\right), v j(\Omega=0)}\left(E_{c}\right)$ is the helicity-resolved ICS. The classical helicity angular momenta $\Omega$ and $\Omega^{\prime}$ are, respectively, the projections of $j$ and $j^{\prime}$ (or equivalently $\boldsymbol{J}$ and $\boldsymbol{J}^{\prime}$ ) onto the reactant and product relative momentum vectors, $\boldsymbol{k}$ and $\boldsymbol{k}^{\prime}$. The helicity-resolved DCS $\sigma_{v^{\prime} j^{\prime}\left(\Omega^{\prime}=0\right), v j(\Omega=0)}\left(\theta, E_{c}\right)$ can be formally written as

$$
\begin{aligned}
& \sigma_{\nu^{\prime} j^{\prime}\left(\Omega^{\prime}=0\right), \nu j(\Omega=0)}\left(\theta, E_{c}\right) \\
& \quad=\frac{1}{k^{2} \sin \theta} \int_{0}^{l_{\max }} P_{\nu^{\prime} j^{\prime}\left(\Omega^{\prime}=0\right), \nu j(\Omega=0)}\left(\theta, l, E_{c}\right) l d l .
\end{aligned}
$$

The computation of the above integrand can be done in a similar way as in Eq. (5). The state-to-state ICSs $\sigma_{v^{\prime} j^{\prime} \Omega^{\prime}, v j \Omega}\left(E_{c}\right)$ for any value of the helicity angular momenta $\Omega$ and $\Omega^{\prime}$ are recovered from the DCSs by integrating over the scattering angle as follows:

$$
\sigma_{\nu^{\prime} j^{\prime} \Omega^{\prime}, \nu j \Omega}\left(E_{c}\right)=2 \pi \int_{0}^{\pi} \sigma_{\nu^{\prime} j^{\prime} \Omega^{\prime}, \nu j \Omega}\left(\theta, E_{c}\right) \sin \theta d \theta,
$$

Details on the derivation and computation of Eqs. (5)-(8) can be found in the Appendix.

Within the present semiclassical PST approach, the diatom vibrational motions are quantized while rotational and translational motions are classically treated. The diatoms are treated as rigid rotor anharmonic oscillators (see Appendix), and the interfragment interaction potential is approximated by a long-range isotropic dispersion term of the form

$$
V(R)=-\frac{C_{6}}{R^{6}},
$$

where the $C_{6}$ coefficient is derived from the SlaterKirkwood formula ${ }^{45}$ for each reactant and product arrangements. Such an isotropic dispersion contribution is known to dominate over the anisotropic electrostatic quadrupolequadrupole term in the long-range part of the $\mathrm{Si}-\mathrm{O}_{2}$ interaction potential. ${ }^{3}$ It is worth noticing that an extension of PST has been recently proposed to approximately account for anisotropic interactions in the asymptotic channels. ${ }^{46}$

\section{RESULTS}

\section{A. Total integral cross sections}

We report in Fig. 1 the total ICSs yielded by the QCT and statistical approaches as a function of collision energy, for three selected $\mathrm{O}_{2}$ rotational states, $j=1,7$, and 13. Selected values of the ICSs are also displayed in Table I. As can be seen in Fig. 1, whatever the rotational state considered is, the QCT cross sections display no energy threshold and are monotically decreasing functions of collision energy. This is the expected feature for an exoergic reaction driven by a barrierless PES, ${ }^{9}$ the reactivity being mainly governed in such a case by the ability of the reactants to overcome the centrifugal energy barrier. The remarkably good agreement of the total ICSs predicted by both SQM and PST approaches with the QCT results can be understood in this sense. Except at the two lowest energies considered (5 and $10 \mathrm{meV}$ ), the SQM and PST ICSs agree with those yielded by QCT calculations within about $2 \%$ and $10 \%$, respectively. This clearly shows that the strong interaction region of the PES does not induce recrossing of the centrifugal barrier towards the reactants through dynamical effects. This is due to the large exo- 


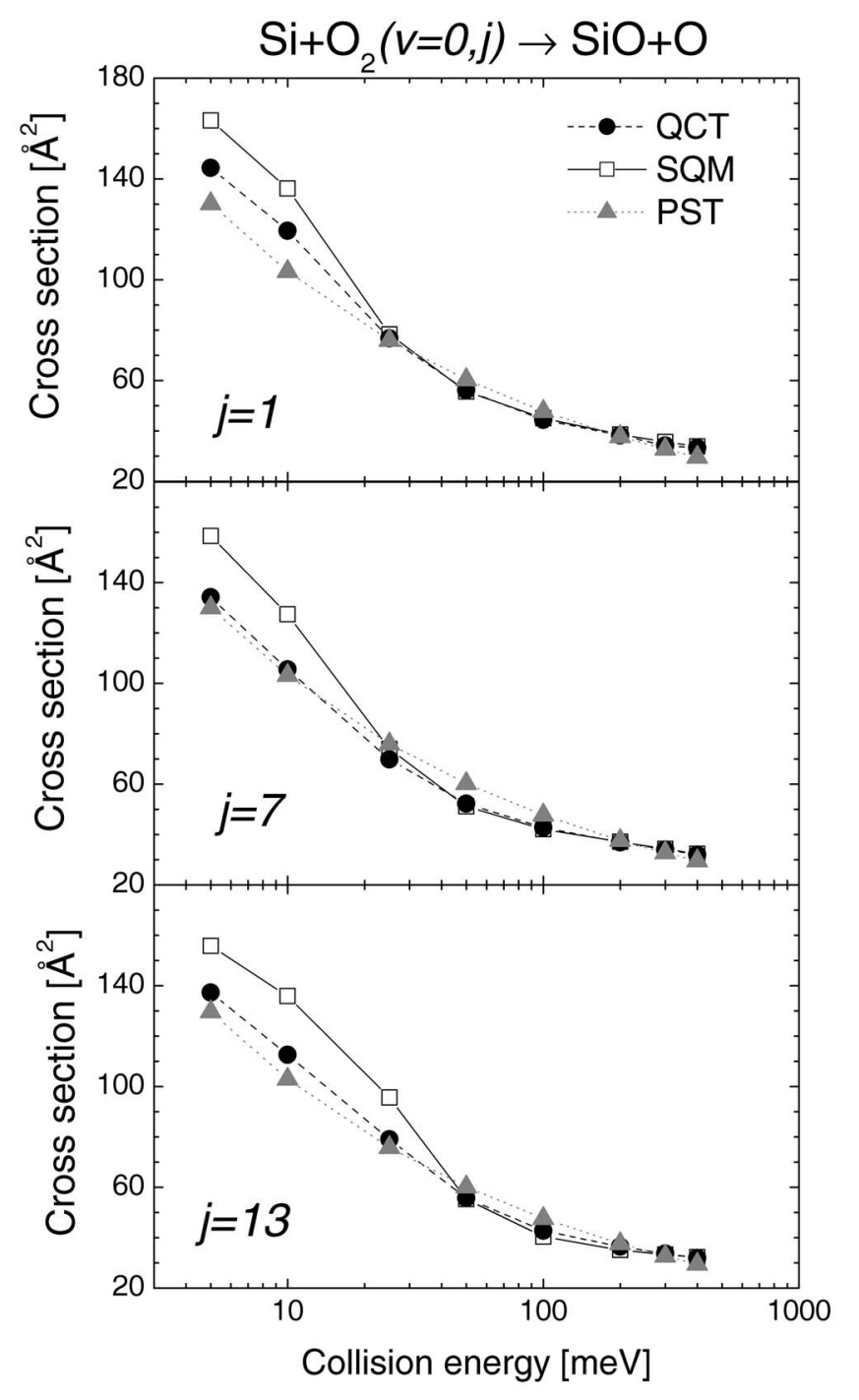

FIG. 1. ICS (in $\AA^{2}$ ) as a function of collision energy (in $\mathrm{meV}$ ) for the $\mathrm{Si}$ $+\mathrm{O}_{2}(v=0, j=1,7,13) \rightarrow \mathrm{SiO}+\mathrm{O}$ reaction computed by means of the QCT (black circles), SQM (open squares), and PST (gray triangles) approaches. The abcissas are shown in logarithmic scale.

ergicity of the reaction, which leads the complex to dissociate almost entirely into products. Moreover, the agreement between SQM and QCT results at energies beyond $25 \mathrm{meV}$ demonstrates the negligible role played by quantum effects in the capture process, as it may be expected for such heavy colliding fragments.

The ICSs values computed with both QCT and SQM approaches display a small but non-negligible dependence with the diatom rotational excitation, especially at low collision energies where the ICSs show an oscillatory feature with $j$ (not shown here) and can vary as much as $15 \%$ accord with the initial rotational state $j$ selected. Such a feature relates to the anisotropic character of the $a b$ initio PES, which favors collinear approaches of the fragments ${ }^{1,8}$ and extends up to rather large interfragment separations. As a matter of fact, the change with $j$ of the ICSs is nearly totally absent from PST predictions, where the calculations of the capture cross sections rest on an isotropic $\mathrm{Si}-\mathrm{O}_{2}$ interaction potential. However, the effect is rather small and should be blurred on average when the cumulative reaction probability or re-
TABLE I. Values of the ICS (in $\AA^{2}$ ) for selected values of collision energy $E_{c}$ (in $\mathrm{meV}$ ) and rotational states $j=1,7,13$ of the $\mathrm{O}_{2}(v=0, j)$ diatomic molecule, obtained by means of the QCT, SQM, and PST methods.

\begin{tabular}{|c|c|c|c|}
\hline$E_{c}(\mathrm{meV})$ & QCT & SQM & PST \\
\hline \multicolumn{4}{|c|}{$j=1$} \\
\hline 5 & 144.4 & 163.2 & 130.2 \\
\hline 100 & 44.4 & 45.0 & 47.7 \\
\hline 400 & 33.3 & 33.8 & 29.6 \\
\hline \multicolumn{4}{|c|}{$j=7$} \\
\hline 5 & 134.2 & 158.6 & 130.0 \\
\hline 100 & 42.7 & 42.1 & 47.7 \\
\hline 400 & 32.0 & 32.3 & 29.6 \\
\hline \multicolumn{4}{|c|}{$j=13$} \\
\hline 5 & 137.3 & 155.8 & 129.7 \\
\hline 100 & 42.8 & 40.4 & 47.6 \\
\hline 400 & 32.0 & 32.2 & 29.5 \\
\hline
\end{tabular}

action rate constant is considered. In this sense, the agreement within $10 \%$ over the whole collision energy range of PST predictions with respect to QCT and SQM results can be regarded as remarkable given the simplicity of the model. According to the isotropic dispersion contribution accounted for in the PST calculations, the total ICS for the $\mathrm{Si}+\mathrm{O}_{2}$ reaction can, thus, up to a certain extent be well approximated by a simple capture cross section varying as $E_{c}^{-1 / 3}$ with collision energy. ${ }^{9}$

As can be seen in Fig. 1 and Table I, the main discrepancies between the different approaches occur below $25 \mathrm{meV}$, where the SQM cross sections are systematically found higher than the QCT ones by about 15\%, independently of the initial $j$ state. Pure quantum effects such as tunneling could be invoked as the origin of those discrepancies. However, both the heavy atom system considered and the expected large width of the centrifugal energy barrier below $25 \mathrm{meV}$ suggest a small influence of this effect. To get additional clues, the discrepancies will be further discussed below in terms of the opacity functions used in the determination of the ICSs.

\section{B. Opacity functions}

We report in Fig. 2 the reaction probability $P_{r}(J)$ as a function of the total angular momentum $J$-hereafter called opacity function-obtained from the QCT, SQM, and PST calculations at selected collision energies and for the $j=1$ case. The results obtained at higher $j$ values have been found to be highly similar to those reported in Fig. 2 and, consequently, they are not shown here. As can be seen in Fig. 2, whatever the collision energy is, the opacity functions provided by the three methods are roughly described by steplike functions with a steric factor close to unity. These features are consistent with the capture model supported by the total ICSs of Fig. 1 and indicate a contribution to reactivity of almost the whole range of molecular configurations up to a maximal angular momentum $J_{\max }$, beyond which no reactive events are found.

Nonetheless, deviations from this simple picture are observed at high $J$ values, where the QCT and SQM opacity 


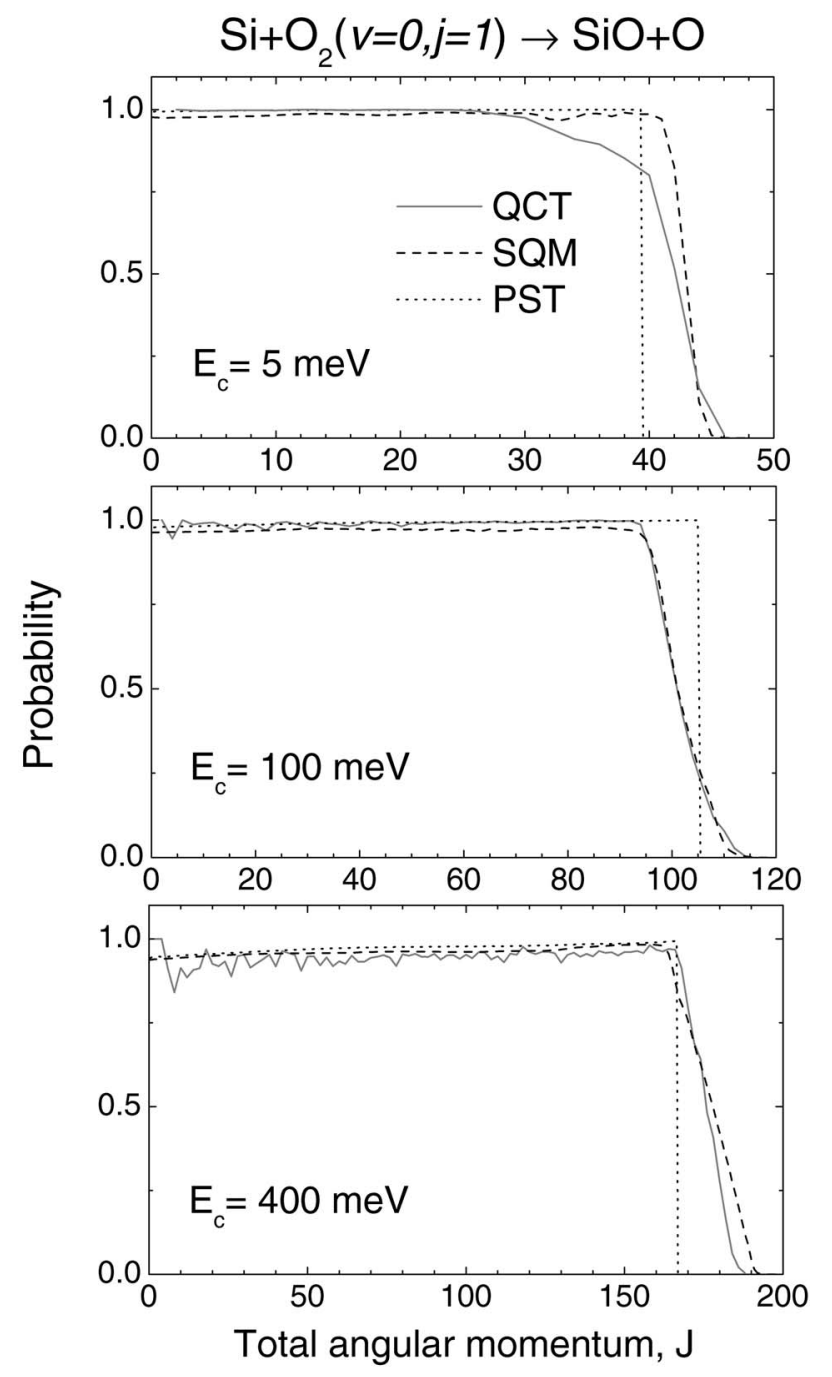

FIG. 2. Reaction probabilities $P_{r}(J)$ as functions of the total angular momentum $J$ for the $\mathrm{Si}+\mathrm{O}_{2}(v=0, j=1)$ reaction at three selected collision energies, obtained by means of the QCT (gray solid line), SQM (dashed line), and PST (dotted line) approaches.

functions $P_{r}(J)$ monotically decrease with $J$ from a given cutoff value $J_{\text {cut }}$ up to $J_{\text {max }}$. The tail of each opacity function maps an almost constant range of angular momentum values $J_{\max }-J_{\text {cut }}$, independently of the selected collision energy. A similar feature is observed when the diatom $\mathrm{O}_{2}$ is further rotationally excited, but the tail of $P_{r}(J)$ maps an increasing range of $J_{\max }-J_{\text {cut }}$ as the selected state $j$ augments. Those features may be understood in terms of the anisotropic character of the PES, not accounted for within the PST approach. Indeed, the $\mathrm{Si}-\mathrm{O}_{2}$ interaction potential corresponding to a collinear approach of the fragment extends on a longer range and falls off earlier than in the case of a perpendicular approach. ${ }^{1,8}$ For near head-on collisions, associated with small values of the orbital angular momentum $|J-j| \leqslant l \leqslant J$ $+j$, the whole range of configurations contributes to reactivity owing to the absence of a potential energy barrier whatever the angle of approach is. However, for collisions characterized by large $l$ values, the anisotropy may play a role on reactivity since, in such a case, the ability of the interaction potential to capture the silicon atom depends on the orientation of the fragments. Thus, the tail of the QCT and SQM opacity functions in Fig. 2 can be viewed in terms of the continuously increasing range of configurations unable to drive the reaction when $J>J_{\text {cut }}$. The wider range of $J_{\max }$ $-J_{\text {cut }}$ observed when the diatom is further rotationally excited can be understood from the decreasing rotational period of the diatom, which reduces the probability to encounter favorable close-to-collinear configurations between the reactants.

The results displayed in Fig. 2 indicate that, except at the lowest energy considered, the QCT and SQM opacity functions are in quite good agreement. Owing to the quantum mechanical nature of the SQM, the close accord with QCT results regarding the tail of the opacity functions indicates that tunneling through the centrifugal barrier is quite negligible. Moreover, the absence of net oscillations in the SQM probabilities suggests that the resonant features are almost completely blurred by the high density of states accessible, what reinforces the use of a classical treatment for the present reaction. Notice that the non-negligible deviations which appear at low $J$ values in the high collision energy range (typically above $100 \mathrm{meV}$ ) are due to a poorer statistic of the QCT opacity functions (see Sec. II A).

Below $25 \mathrm{meV}$, the QCT and SQM reaction probabilities significantly differ at large $J$ values. If one considers the degeneracy factor $2 J+1$ involved in the partial wave average of Eq. (3), it clearly appears that such a deviation of the reaction probabilities is at the origin of the discrepancies observed below $25 \mathrm{meV}$ between the QCT and SQM total ICSs (see Fig. 1). Similar discrepancies between the SQM and QCT results have already been observed for the final state-resolved opacity functions associated with the $\mathrm{C}+\mathrm{H}_{2}$, $\mathrm{H}^{+}+\mathrm{H}_{2}$, and $\mathrm{H}^{+}+\mathrm{D}_{2}$ reactions. ${ }^{17,47,48}$ The results obtained by means of the SQM approach in its present CS version have been compared to those yielded by the SQM approach in its close-coupling (CC) and QCT (Ref. 16) versions in the case of $\mathrm{C}+\mathrm{H}_{2}$ and those yielded by QCT and EQM calculations in the $\mathrm{H}^{+}+\mathrm{H}_{2}$ and $\mathrm{H}^{+}+\mathrm{D}_{2}$ cases. A close inspection of the results suggests that the use of the CS approximation can be responsible for non-negligible deviations of the state-to-state opacity functions at large $J$. Although the latter deviations are largely reduce by summing over all final states, the very low collision energy regime considered here could reinforce such a failure of the CS approximation. We, thus, performed test calculations at $5 \mathrm{meV}$ and $j=1$ by using the SQM approach in its $\mathrm{CC}$ version. Although the $J_{\max }=45$ value could not be reached due to the huge number of states to account for, the SQM-CC opacity function agrees nicely with the SQM-CS values up to $J=38$, where the QCT and SQM-CS results strongly deviate (Fig. 2). It, thus, appears that the most likely explanation to the discrepancy observed at large $J$ below $25 \mathrm{meV}$ rests on a failure of the QCT approach. Following a recent proposal by Bonnet, ${ }^{49}$ this could be due to an overestimation of the weight associated with vibrationally (quasi)adiabatic nonreactive trajectories in the QCT treatment. Those events are expected to predominantly occur when the trajectories are trapped at large interfragment separations, in a potential well region where the coupling between the vibrational motion and the remaining ones are small. Since low collision energies and large angular mo- 


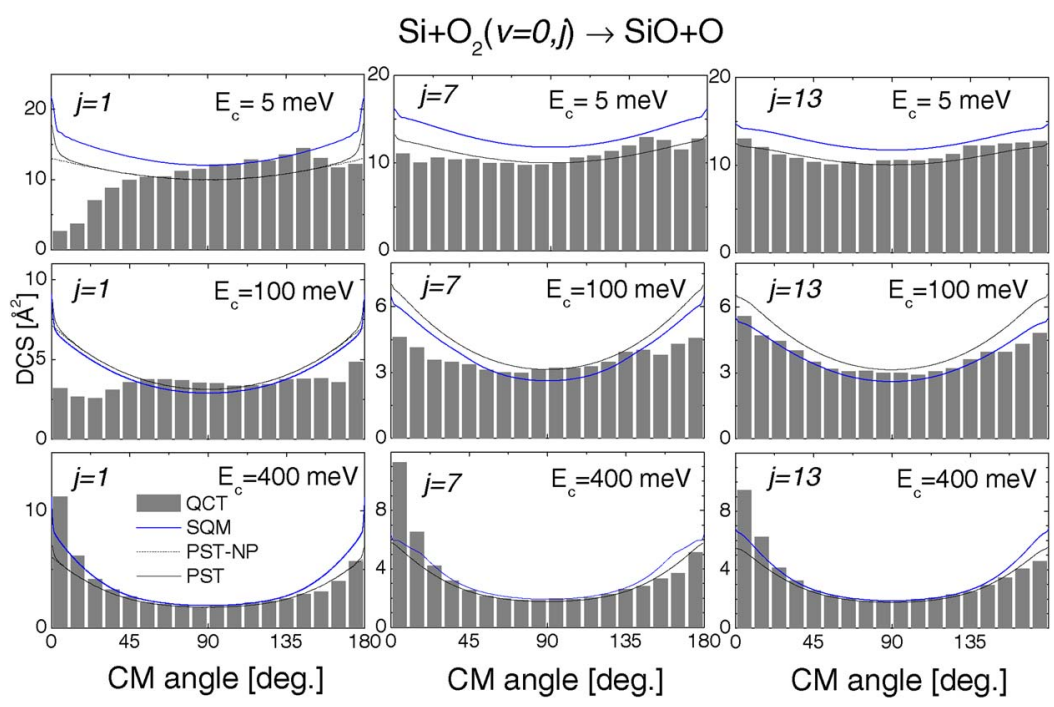

FIG. 3. (Color online) Total DCSs for the $\mathrm{Si}+\mathrm{O}_{2}$ $(v=0, j)$ reaction at selected collision energies and rotational states $j=1,7,13$, obtained by means of the QCT (histogram), SQM (solid blue line), and PST including (solid black line) or not (dashed line) parity correction.

mentum values may favor such a situation, the bias toward inelastic events in the QCT treatment should significantly reduce the reaction probability at low $E_{c}$ and large $J$ values, as it is found in the present case.

\section{Differential cross sections}

A deeper insight on the reaction dynamics is provided by the total DCSs calculated by means of the QCT, SQM, and PST-including or not the parity correctionmethodologies. The results are shown in Fig. 3 for selected values of the collision energy and rotational angular momentum of the reactant diatom. From the results of QCT calculations, it is readily seen that the latter two initial conditions strongly affect the resulting DCSs. At low collision energy and low rotational excitation of $\mathrm{O}_{2}$, the QCT DCSs display a well-marked preference for backward scattering, as it is shown by the mean scattering angle value $\langle\theta\rangle=97^{\circ}$ and the forward/backward ratio $f / b=0.17$ recorded at $E_{c}=5 \mathrm{meV}$ for the $j=1$ case. Such a feature is consistent with the dominant contribution of the direct abstraction mechanism outlined in a previous study of the $j=1$ case. $^{8}$ Indeed, the linear geometry of the $\mathrm{SiOO}$ short-lived intermediate complex MIN1 involved during the bond exchange tends to produce backward scattered products through a rebound mechanism. As a result of such a direct mechanism, a tight correlation between the initial impact parameter $b$ and the angular distribution is found, where low $b$ values lead to products preferentially scattered in the backward hemisphere, whereas high $b$ values result in a dominant forward scattering. The backward character of the resulting DCS arises from the broad range of impact parameter values leading to backward scattering (typically $b<5 \AA$ while $b_{\max }=7.2 \AA$ ), hence, providing further evidences of the ability of the PES to drive the reactants toward the linear $\mathrm{SiOO}$ geometry at low collision energies. This feature of the DCSs observed at low $E_{c}$ and $j$ values, strongly related to the constrained geometry of the short-lived intermediate, can obviously not be described by the statistical treatments, whose underlying assumptions imply completely forward/backward symmetric DCSs.
By contrast, the increase in either the collisional energy or rotational excitation of $\mathrm{O}_{2}$ results in QCT DCSs with a near symmetrical forward/backward profile. Consequently, the agreement with statistical predictions is considerably improved, as can be seen in Fig. 3. The discrepancies observed despite the near symmetrical forward/backward profile of the QCT DCSs, particularly at low $E_{c}$ and high $j$ values, have to be viewed in terms of the distinct ICSs yielded by the three methodologies (see Sec. III A). Furthermore, the DCSs computed from the two PST treatments provide additional evidences of the need of a parity restoring correction to get a correct description of the extreme forward/backward peaks of the DCSs, the improvement being quite noticeable at low collision energies (see Fig. 3).

The statistical feature displayed by the DCSs at high collision energies was expected in the $j=1$ case given the dominant insertion mechanism involving two long-lived (symmetric) intermediates, the bent and linear OSiO species MIN2 and MIN3, which had been brought to evidence. ${ }^{8}$ The similar feature observed on increasing $j$ values suggests that the rotational excitation has little effect on the reaction mechanism at high collision energies. Nonetheless, it is worth noticing that, despite the dominant insertion mechanism, nonstatistical feature persists in the highest collision energy range, where the QCT DCSs exhibit a slight asymmetry with a maximum peak in the forward direction $\left(\langle\theta\rangle=86^{\circ}\right.$ and $f / b=1.97$ for $E_{c}=400 \mathrm{meV}$ and $\left.j=1\right)$. This feature may be related to a remaining contribution of the direct abstraction mechanism. What is more striking is the coincidence between the QCT and statistical DCSs obtained at the lowest collision energies when the diatom is rotationally excited. The QCT DCSs exhibit a symmetrical forward /backward profile $\left(\langle\theta\rangle=91^{\circ}\right.$ and $f / b=1.02$ for $E_{c}=5 \mathrm{meV}$ and $j=13)$ in close agreement with statistical predictions. Consistently, the correlation between the impact parameter $b$ and the scattering angle is found to be much weaker on increasing $j$ values. Those findings indicate that, even at the lowest energy probed, the high rotational excitation of $\mathrm{O}_{2}$ leads the reaction to proceed through an intermediate complex whose lifetime is longer than its rotational period. This strongly suggests that the rotational excitation and the colli- 


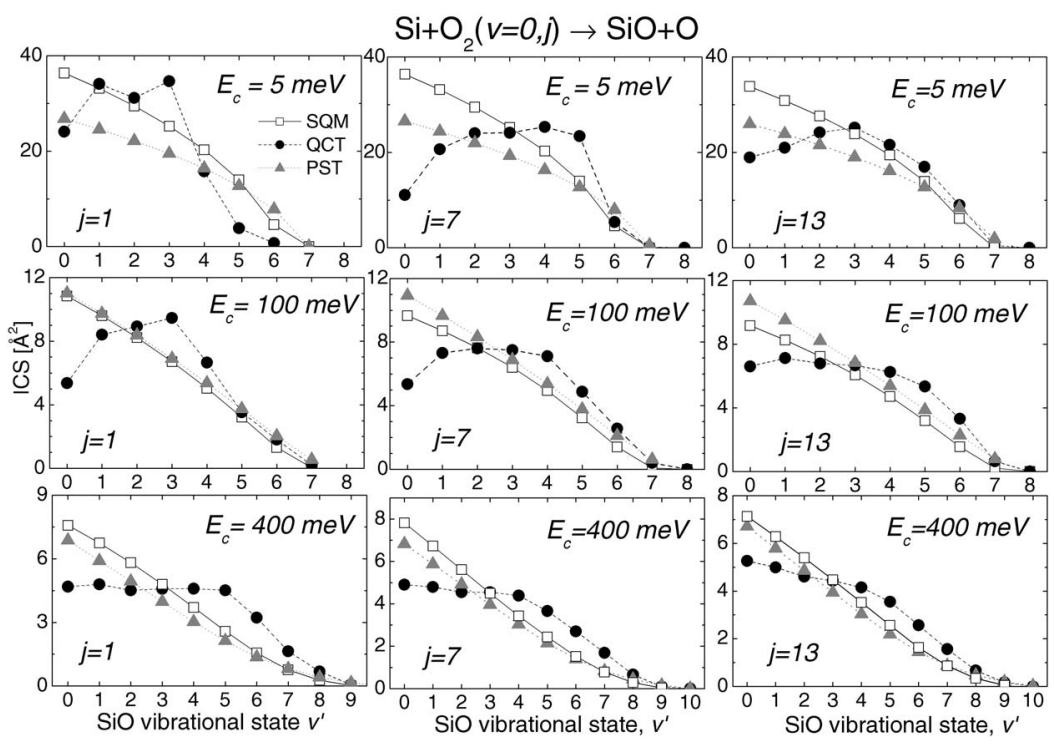

FIG. 4. Final vibrational-state-resolved cross sections for the $\mathrm{Si}+\mathrm{O}_{2}(v=0, j) \rightarrow \mathrm{SiO}\left(v^{\prime}\right)+\mathrm{O}$ reaction at selected values of the collision energy and rotational angular momentum $j$, obtained by means of the QCT (solid line), SQM (dashed line), and PST (dotted line) approaches.

sional energy affect in a similar manner the reaction mechanisms at work, leading to a continuous transition from abstraction to insertion mechanisms. This point will be further discussed in Sec. IV.

Regarding the shape of the DCSs, it is quite remarkable that both the SQM and PST statistical treatments predict a polarization of the DCSs in close agreement with QCT results. This is readily seen for the DCSs obtained at high $j$ values, where a noticeable enhancement of forward/ backward scattering at the expense of sideway scattering is yielded by the three methodologies on increasing the collision energy. Such a close agreement between QCT and statistical results points out that this effect rests on kinematic constraints on the angular momentum disposal in the reactant and product channels, instead of having a dynamical origin. By following previous statistical models, ${ }^{4,50}$ a rough estimate of the angular momentum partitioning has been derived from QCT calculations of the scalar quantities $\Lambda=\langle l /(l+j)\rangle$ and $\Lambda^{\prime}=\left\langle l^{\prime} /\left(l^{\prime}+j^{\prime}\right)\right\rangle$ associated with the reactant and product arrangements, respectively. Likewise, the averaged quantity $\left\langle\cos ^{2} \theta\right\rangle$, where the c.m. scattering angle $\theta$ corresponds to the $\mathbf{k}-\mathbf{k}^{\prime}$ angular correlation, has also been computed. For $E_{c}$ $=5 \mathrm{meV}$ and the $j=13$ case, we found $\Lambda=0.64$ and $\Lambda^{\prime}$ $=0.51$. Since $J_{\max }$ is quite small with respect to the populated $j^{\prime}$ states, it can be considered that $\mathbf{l}^{\prime}$ and $\mathbf{j}^{\prime}$ have almost the same modulus and opposite direction. In such a case, one expects an isotropic angular distribution in the collision plane and, hence, an isotropic DCS. The DCS displayed in Fig. 3, as well as the computed value $\left\langle\cos ^{2} \theta\right\rangle=0.34$, close to $1 / 3$, are consistent with this prediction. For $E_{c}=400 \mathrm{meV}$ and the $j=13$ case, we found $\Lambda=0.88$ and $\Lambda^{\prime}=0.62$. Since at high collision energies, $J_{\max }$ is large with respect to the populated $j^{\prime}$ states, we are close to the limiting case $l^{\prime}>j^{\prime}$ and $\mathbf{l}^{\prime} \approx \mathbf{J}$. The statistical models predict in such a case a strongly peaked DCS, approaching the $1 / \sin \theta$ behavior. The DCS of Fig. 3 and the computed value $\left\langle\cos ^{2} \theta\right\rangle=0.44$, close to $1 / 2$, show this is, indeed, the case.

\section{Product energy distribution}

Detailed information about the energy partitioning into the products modes is provided by the final state-resolved cross sections computed from the QCT, SQM, and PST methodologies. The product vibrational $\mathrm{SiO}\left(v^{\prime}\right)$ and rotational $\mathrm{SiO}\left(v^{\prime}=0, j^{\prime}\right)$ distributions obtained for selected values of the collision energy and rotational angular momentum $j$ are shown in Figs. 4 and 5, respectively. Likewise to the DCSs previously discussed, the QCT product state distributions are found to be strongly modified according to the selected initial conditions of the reaction. As can be seen in Fig. 4, the QCT vibrational distribution obtained at $E_{c}=5 \mathrm{meV}$ for the $j=1$ case clearly departs from the statistical results. The QCT distribution shows a noticeable vibrational inversion, peaked at $v^{\prime}=3$, with the states $\operatorname{SiO}\left(v^{\prime}=0\right)$ and $\mathrm{SiO}\left(v^{\prime} \geqslant 5\right)$ being significantly less populated than both statistical predictions. Notice that the SQM and PST statistical approaches predict highly similar vibrational distributions over the whole range of energies and accessible $v^{\prime}$ states, except below $25 \mathrm{meV}$ for $\mathrm{SiO}\left(v^{\prime}<5\right)$. Such a close agreement on fine details of the product state properties is quite remarkable given the underlying approximations to the PST approach. From the comparison between the QCT and statistical rotational distributions shown in Fig. 5 for $E_{c}=5 \mathrm{meV}$ and $j=1$, a similar well-marked nonstatistical feature emerges. The QCT rotational distribution is peaked at around $j^{\prime}=10$ (almost independently of $v^{\prime}$ ) and is much colder than its statistical counterparts, which exhibit significant population up to $j^{\prime} \approx 100$, whereas the QCT distribution ends at about $j^{\prime}=30$. Such a specific energy release into the rovibrational product modes is consistent with the direct abstraction mechanism outlined before: ${ }^{8}$ The constrained linear geometry of the $\mathrm{SiOO}$ intermediate tends to expel the $\mathrm{SiO}$ molecule with low rotational excitation, and the sudden mechanism favors a limited range of product vibrational states.

As can be seen in Figs. 4 and 5, the situation dramatically changes on increasing either the collision energy or the rotational angular momentum $j$ of the reactant diatom. The vibrational inversion is gradually attenuated in the QCT vibrational distributions, which become nearly monotonic decreasing functions of $v^{\prime}$ in a much closer agreement with the SQM and PST statistical profiles. Nonetheless, even at the largest $E_{c}$ and $j$ values, the QCT vibrational distributions 


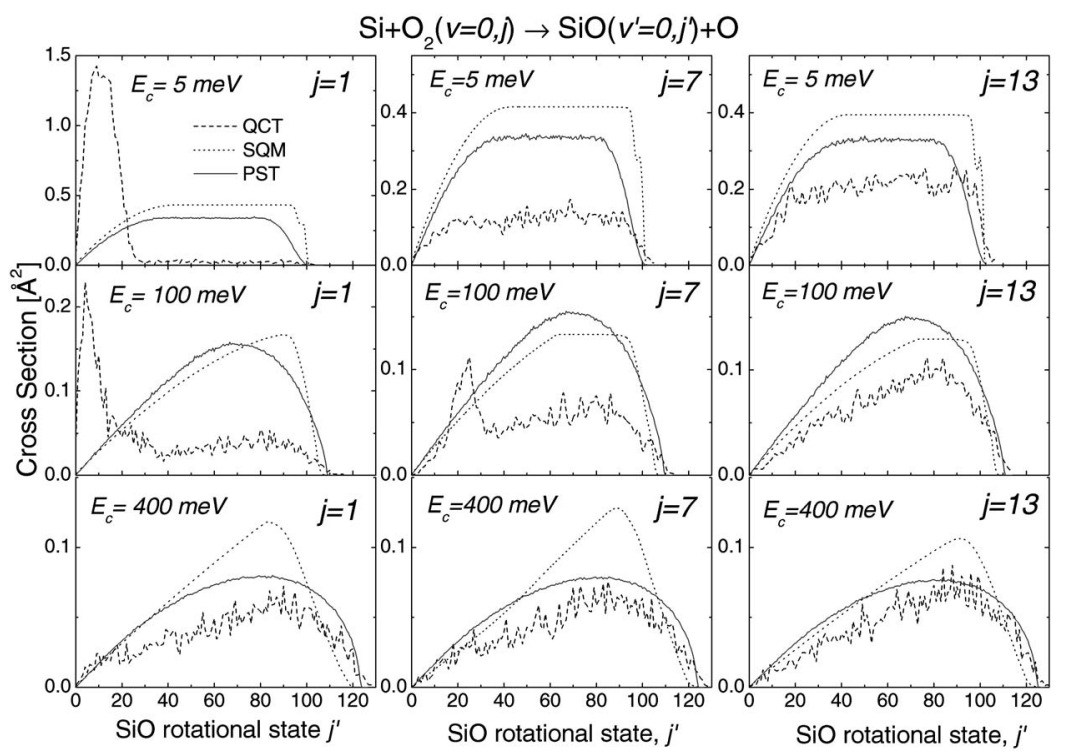

FIG. 5. Final rotational-state-resolved cross sections for the $\mathrm{Si}+\mathrm{O}_{2}(v=0, j) \rightarrow \mathrm{SiO}\left(v^{\prime}=0, j^{\prime}\right)+\mathrm{O}$ reaction at selected values of the collision energy and rotational angular momentum $j$, obtained by means of the QCT (dashed line), SQM (dotted line), and PST (solid line) approaches.

remain to be somewhat hotter than the statistical ones, with the states $\operatorname{SiO}\left(v^{\prime}>3\right)$ being slightly more populated than statistical predictions, and reversely for $\mathrm{SiO}\left(v^{\prime}<3\right)$. Besides, the results of Fig. 4 suggest a decreasing influence of the rotational excitation of $\mathrm{O}_{2}$ on increasing collision energy.

In a similar way, the QCT rotational distributions depicted in Fig. 5 for $\mathrm{SiO}\left(v^{\prime}=0\right)$ indicate a clear tendency for the reaction to populate the final rotational $j^{\prime}$ states in a statistical manner on increasing either the collision energy or the rotational angular momentum $j$ of the reactant diatom. In the $j=1$ case, the prominent maximum peak obtained at low $j^{\prime}$ states for $E_{c}=5 \mathrm{meV}$ tends to be soften on increasing $E_{c}$ at the expense of a non-negligible population in the higher rotational states. This gives rise to bimodal rotational distributions, up to the highest collision energy range, where the QCT rotational distribution is found in a qualitatively good agreement with both statistical profiles. Similar comments hold from the results obtained at fixed collision energies, the production of rotationally cold $\mathrm{SiO}$ molecules gradually decreasing on increasing the rotational excitation of $\mathrm{O}_{2}$. A noticeable exception to this feature concerns the $j=7$ case at $E_{c}=5 \mathrm{meV}$, where the QCT rotational distribution displays an almost statistical profile, in clear contrast to the bimodal feature obtained at $E_{c}=100 \mathrm{meV}$. A close inspection of the QCT results at $E_{c}=5 \mathrm{meV}$ and $j=7$ told us that, in fact, the rotational distributions associated with the $\mathrm{SiO}\left(v^{\prime}>0\right)$ states (not shown here) display a prominent peak at around $j^{\prime}$ $=20$, totally absent from statistical predictions. Interestingly, this finding is found to be quite general, the $\operatorname{SiO}\left(v^{\prime}>0, j^{\prime}\right)$ rotational distributions displaying a nonstatistical character much more marked than it is found for $\operatorname{SiO}\left(v^{\prime}=0\right)$, with a predominant production of rotationally cold $\mathrm{SiO}$ molecules. Such a tendency is observed, although to a much lesser extent, even in the extreme case considered, says, the $j=13$ case at $E_{c}=400 \mathrm{meV}$.

\section{DISCUSSION}

\section{A. Reaction mechanisms}

To get a deeper insight into the reaction mechanisms, we have discriminated the reactive trajectories obtained from
QCT calculations according to the potential well regions explored during the reaction. The abstraction mechanism labels the trajectories passing only through the linear SiOO intermediate, MIN1, whereas those passing through the bent and linear OSiO intermediates, MIN2 and MIN3, respectively, are classified as pertaining to the insertion mechanism. Details on the procedure employed to discriminate the trajectories can be found in Ref. 8. We report in Fig. 6 the contribution to reactivity of both reaction mechanisms as a function of collision energy, for the three selected rotational states of the $\mathrm{O}_{2}$ diatom, $j=1,7$, and 13 . In the $j=1$ case, the abstraction mechanism is found to be the main reaction mechanism below $25 \mathrm{meV}$, whereas on increasing $E_{c}$, its contribution gradually diminishes at the expense of insertion, which becomes the dominant mechanism above $250 \mathrm{meV}$. Likewise, in the low energy regime, the contribution to reactivity of the abstraction mechanism diminishes at the expense of insertion on increasing the rotational excitation, the insertion mechanisms having the dominant contribution at $5 \mathrm{meV}$ for $j \geqslant 9$. The influence of rotational excitation onto the reaction mechanisms tends to diminish with $E_{c}$ and becomes almost negligible in the highest collision energy range. Interestingly, for each of the rotational states $j \geqslant 9$, we found a short range of energies for which the contribution of abstraction increases with $E_{c}$.

The abstraction-to-insertion transition which comes along the increases in $E_{c}$ in the $j=1$ case can be readily understood in terms of the decreasing influence of the longrange part of the PES, whose anisotropic character drives the reactants into close to collinear $\mathrm{SiOO}$ configurations and, hence, to MIN1, at low collision energies. ${ }^{8}$ Such a feature of the capture process is uncovered by the continuous decrease of the maximum impact parameter $b_{\max }$ value with $E_{c}$, which changes from 7.2 to $3.5 \AA$ between 5 and $400 \mathrm{meV}$ in the $j=1$ case. The abstraction-to-insertion transition observed on increasing $j$ at low $E_{c}$ values cannot be explained in those terms. Indeed, the increase in the total energy with $j$ is rather small (about $30 \mathrm{meV}$ from $j=1$ to 13 ) and the $b_{\max }$ values are found to be almost independent of $j$. 


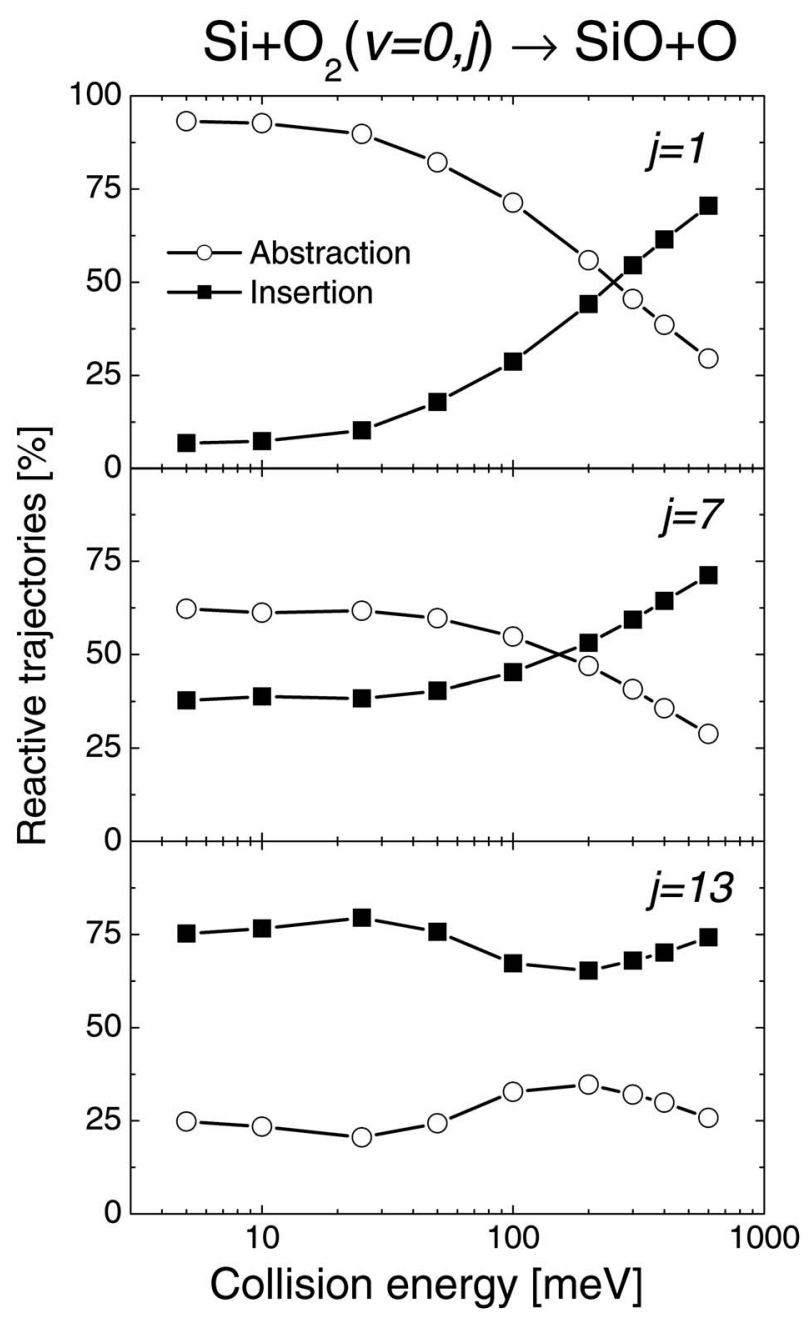

FIG. 6. Contributions to reactivity of the insertion (empty circles) and abstraction (solid squares) reaction mechanisms for the $\mathrm{Si}+\mathrm{O}_{2}(v=0, j)$ reaction, where $j=1,7$, and 13 , as a function of the collision energy. The abcissas are shown in logarithmic scale.

The most likely explanation to such a change of the reaction mechanisms with $j$ rests on the decreasing rotational period $\tau_{\text {rot }}$ of the diatom, which may reduce the probability to form the linear complex MIN1. Indeed, at low collision energy, the $\mathrm{Si}$ atom is captured by the long-range part of the PES preferentially for a collinear orientation of the fragments. The subsequent reaction mechanism, abstraction or insertion, may then vary according to the ratio between the time needed for the diatom to rotate by about $\pi / 2\left(\tau_{\pi / 2}\right)$ and the time needed for $\mathrm{Si}$ to approach the diatom until the complex formation $\left(\tau_{\text {app }}\right)$. A close inspection of a sample of trajectories told us that the elapsed time between a noticeable change of the Si kinetic energy and the formation of the intermediate complex is typically $\tau_{\text {app }}=0.2 \mathrm{ps}$ at $5 \mathrm{meV}$. If one considers $\mathrm{O}_{2}$ as a rigid rotor, the rotational periods of the diatom, $\tau_{\mathrm{rot}}=\pi \hbar / B \sqrt{j(j+1)}$, are found to be equal to 8.2, 1.6, and 0.9 ps for $j=1,7$, and 13 , respectively, corresponding to $\tau_{\pi / 2}=2,0.4$, and 0.2 ps. Neglecting all couplings between translational and rotational motions, the ratio between $\tau_{\text {app }}$ and $\tau_{\pi / 2}$ is, thus, consistent with an enhancement of the insertion mechanism on increasing $j$. Obviously, such a trend tends to disappear on increasing $E_{c}$, given that the capture of
Si for a collinear orientation of the fragments is less and less privileged with respect to other orientations. Besides, it exists a short range of energies specific to each of the rotational states $j \geqslant 9$ where the anisotropy of the PES still have an influence on the capture process and where $\tau_{\text {app }}<\tau_{\pi / 2}$, giving rise to a slight enhancement of the abstraction mechanism with $E_{c}$ (see Fig. 6).

\section{B. Influence on the product state properties}

We report in Fig. 7 the product state properties associated with each reaction mechanism for $E_{c}=100 \mathrm{meV}$ and $j=7$, an intermediate case where both mechanisms have an almost equal contribution to reactivity (Fig. 6). From Fig. 7, it is readily seen that the abstraction leads to a wellmarked bell-shape vibrational distribution, peaked at around $v^{\prime}=4$, a cold rotational distribution, centered around $j^{\prime}=20$, and a total DCS which displays a slight preference for backward scattering. Such features have been found to be rather independent of the selected initial conditions, the most noticeable changes, observed on increasing $E_{c}$ values, being a vibrational distribution shifted to higher $v^{\prime}$ states, a somewhat hotter rotational distribution, and a total DCS peaked in the forward direction due to the inertial effect of the incoming $\mathrm{Si}$ atom. Thus, whatever the initial conditions of the reaction are, the abstraction mechanism yields nonstatistical product state properties, consistently with the constrained linear geometry and the quite short lifetime (of about $15 \mathrm{fs}$ ) associated with the SiOO intermediate involved.

At the opposite, the results of Fig. 7 provide a clear evidence of the statistical product state properties yielded by the insertion mechanism. Indeed, the QCT product distributions are remarkably well described by those obtained from the SQM and PST statistical approaches, and the same agreement has been found for the whole set of initial conditions $\left(E_{c}, j\right)$ probed. The statistical results shown in Fig. 7 have been normalized in such a way to remove the contribution of abstraction from the statistical ICS; i.e., we report $X_{\text {stat. }}^{\prime}$. $=X_{\text {stat. }} \mathrm{ICS}_{\mathrm{QCT}}^{\text {insert. }} / \mathrm{ICS}_{\text {stat. }}$, where $X_{\text {stat. }}$ is a given property (rovibrational distribution, DCS) obtained from the statistical approaches and $\mathrm{ICS}_{\mathrm{QCT}}^{\text {insert. }}=\mathrm{ICS}_{\mathrm{QCT}}-\mathrm{ICS}_{\mathrm{QCT}}^{\text {abstra. }}$. The normalized results $X_{\text {stat. }}^{\prime}$ obtained from SQM and PST calculations are almost indistinguishable at the scale of the figure. The statistical features found for the insertion mechanism are consistent with the long-lived symmetric $\mathrm{OSiO}$ intermediates involved. Indeed, the lifetime of each intermediate is relatively small, of about $60 \mathrm{fs}$, but the $\mathrm{SiO}_{2}$ molecule experiences several transits between the bent and linear $\mathrm{OSiO}$ potential wells during the reaction, giving rise to an effective lifetime of about 0.3 ps for each intermediate. Furthermore, for such an heavy atoms system such as $\mathrm{SiO}_{2}$, the energy randomization may be reinforced by the large coupling strength between the vibrational modes of each complex and the associated large density of states. ${ }^{51}$ 

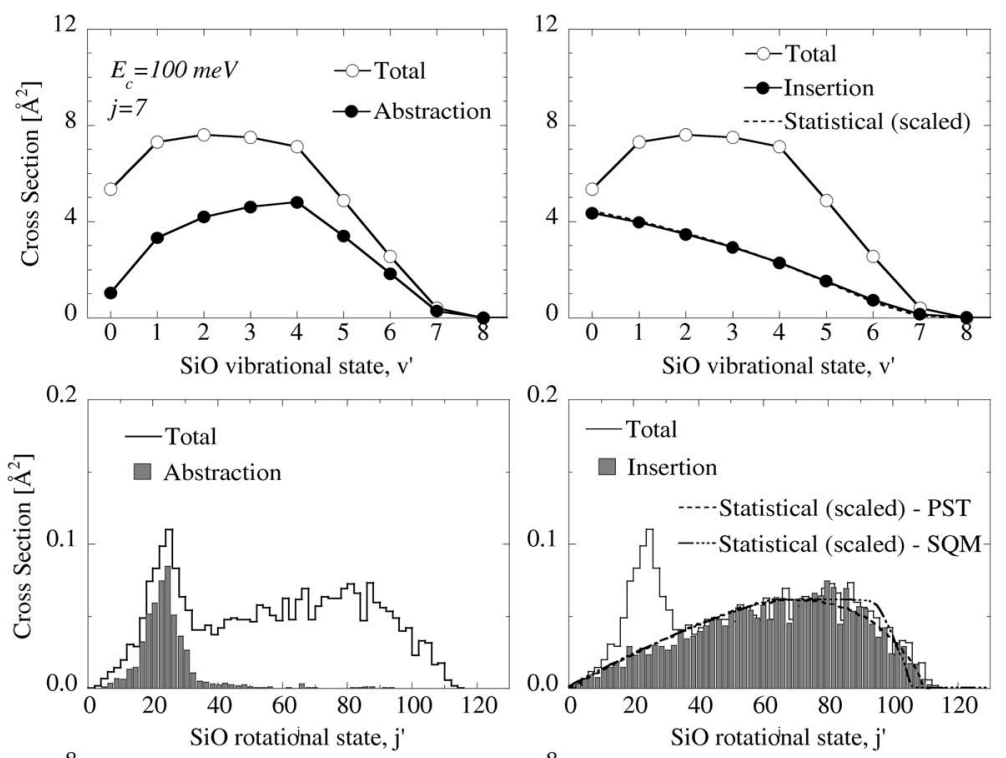

FIG. 7. Product state properties associated with the abstraction and insertion mechanisms for the $\mathrm{Si}+\mathrm{O}_{2}$ $(v=0, j) \rightarrow \mathrm{SiO}+\mathrm{O}$ reaction at $j=7$ and $E_{c}=100 \mathrm{meV}$, obtained by means of QCT calculations. The dashed lines are the results of the SQM and PST approaches (almost indistinguishable at the scale of the figure), normalized in such a way to remove the contribution of abstraction to the statistical ICS. Upper panel: Vibrational distributions $\mathrm{SiO}\left(v^{\prime}\right)$. Middle panel: Rotational distributions $\mathrm{SiO}\left(v^{\prime}=0, j^{\prime}\right)$. Lower panel: Total DCSs.
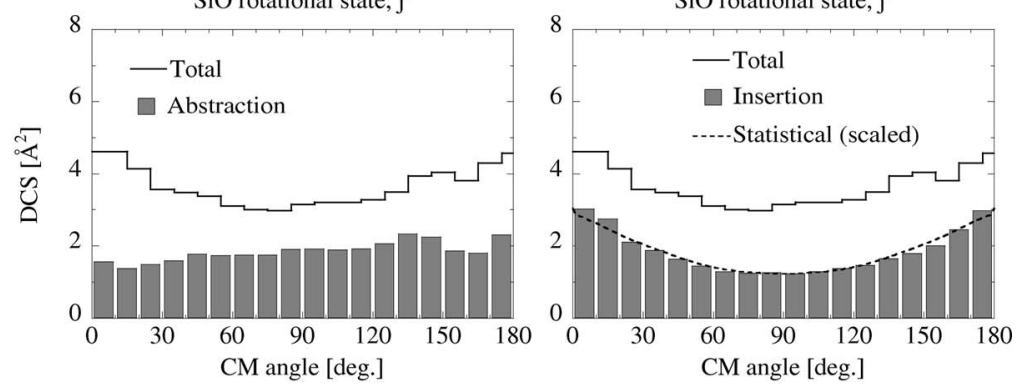

The results of Figs. 6 and 7 clearly show that the changes observed for the product state properties (Figs. 3-5) according to the selected collision energy and $\mathrm{O}_{2}$ rotational excitation can be fully explained in terms of the relative contributions to reactivity of the two reaction mechanisms. Interestingly, a first experimental information about the product state distributions of the title reaction has been recently obtained by Yamashiro et al. by using a crossed-beam laserinduced fluorescence technique. ${ }^{7}$ The collision energy considered in experiment was $135 \mathrm{meV}(13 \mathrm{~kJ} / \mathrm{mol})$, and the rotational temperature of $50 \mathrm{~K}$ for $\mathrm{O}_{2}$ corresponds to a Boltzmann distribution peaked at $j=3$ and ending at around $j=11$. The experimental $\mathrm{SiO}\left(v^{\prime}\right)$ vibrational distribution is displayed in Fig. 8, together with the results of QCT, SQM, and PST calculations carried out under the latter initial conditions. Accordingly with the discussion of Sec. III A, the SQM and PST rotationally averaged ICS agrees with that obtained from QCT calculations $\left(\sigma=40.3 \AA^{2}\right)$ within $2 \%$ and $10 \%$, respectively. Nevertheless, the dominant contribution to reactivity of the abstraction mechanism (about 60\%) results in a QCT bell-shape vibrational distribution which cannot be reproduced by statistical approaches, as can be seen in Fig. 8. The QCT result may explain the inverted vibrational distribution observed in experiment but not the significant populations observed for the $v^{\prime}=0,4$ and $v^{\prime} \geqslant 7$ states. Although the QCT values lie within the range of experimental uncertainties for all states but $v^{\prime}=0$ and 1 , one may also invoke the role of the ${ }^{3} A^{\prime}$ triplet electronic state to explain such discrepancies. Indeed, a previous comparison between experimental and theoretical rate constants at low to room temperatures ${ }^{1}$ suggested a reaction rate constant for the ${ }^{3} A^{\prime}$

state at least equal to that of the ${ }^{1} A^{\prime}$ state and resulting in a much larger contribution to reactivity due to the electronic partition function, $k^{\text {tot }}=(1 / 27)\left[k\left({ }^{1} A^{\prime}\right)+3 k\left({ }^{3} A^{\prime}\right)\right]$. In the present case, no definite conclusions can be drawn on the specific contribution to reactivity of each electronic state, since absolute values of the total ICS or of the $\mathrm{O}\left({ }^{3} P\right) / \mathrm{O}\left({ }^{1} D\right)$ branching ratio are still lacking (assuming $\sigma\left({ }^{3} A^{\prime}\right)=\sigma\left({ }^{1} A^{\prime}\right)$, we would expect $\sigma^{\text {tot }}=6.0 \AA^{2}$ ). However, both the results of

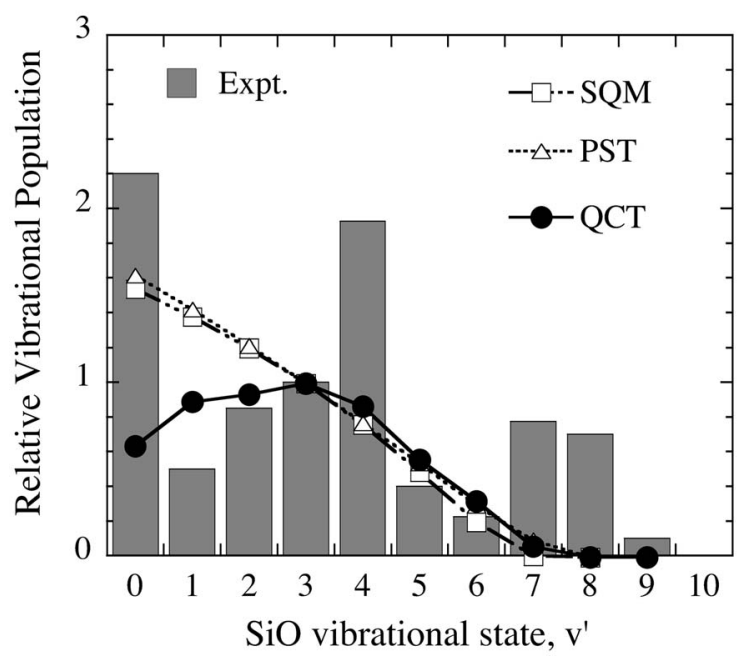

FIG. 8. Final vibrational state distribution of $\mathrm{SiO}\left(v^{\prime}\right)$ for the $\mathrm{Si}+\mathrm{O}_{2}$ reaction, at a collision energy of $135 \mathrm{meV}$ and a rotational temperature of $50 \mathrm{~K}$ for $\mathrm{O}_{2}$. The experimental distribution ${ }^{7}$ is compared with results of QCT (solid line), SQM (solid-dashed line), and PST (dashed line) approaches associated with the singlet channel of the reaction. All distributions have been normalized at $v^{\prime}=3$. 
Fig. 8 and the detection of $\mathrm{O}\left({ }^{3} \mathrm{P}\right)$ in experiment point out to a significant contribution to reactivity of the ${ }^{3} A^{\prime}$ state and possibly proceeding through a direct mechanism owing to the specific population of the $v^{\prime}=4$ and $v^{\prime} \geqslant 7$ states. It is also worth noting that Yamashiro et al. suggested the existence of spin-orbit mixing between the singlet and triplet channels to explain the nonstatistical population detected for the $\mathrm{O}\left({ }^{3} P_{2}\right)$ spin-orbit state. ${ }^{7}$

\section{CONCLUSIONS}

An extensive study of the dynamics associated with the singlet channel of the $\mathrm{Si}+\mathrm{O}_{2} \rightarrow \mathrm{SiO}+\mathrm{O}$ reaction has been undertaken in the present study, by means of QCT calculations and two statistical based methods, the SQM and a semiclassical version of PST, extended to deal with a rotationally excited reactant diatom. It has been found that the total ICSs and opacity functions yielded by the three theoretical methods are in a remarkably good agreement over a wide range of collision energies (from 400 down to $5 \mathrm{meV}$ ), independently of the selected rotational state of the diatomic oxygen molecule (from $j=1$ up to 13). A maximal deviation of about $15 \%$ between the QCT and SQM total ICSs is obtained below $25 \mathrm{meV}$, linked to an underestimation of the reactivity at high $J$ values by the QCT treatment, which is probably due to an overestimated weight of vibrationally (quasi)adiabiatic nonreactive trajectories. At and above $25 \mathrm{meV}$, the SQM and PST total ICSs agree with QCT results within about $2 \%$ and $10 \%$, respectively, pointing out that recrossing effects due to the dynamics in the strong interaction region, as well as tunnel effects, are almost negligible, and that an isotropic longrange dispersion interaction, as it is used in PST calculations, can serve to catch the main reactive features. Such a result is quite encouraging given the difficulty to deal with the whole dynamics of the $\mathrm{Si}+\mathrm{O}_{2}$ reaction, in particular, by means of EQM calculations. As regards the product state properties, the agreement between the three methodologies has been found to greatly vary according to the selected initial conditions. Indeed, the increase in either the collision energy or the $\mathrm{O}_{2}$ rotational excitation strongly modifies the main reaction pathway, leading to a continuous transition from abstraction to insertion mechanisms. As a result of the direct character of the abstraction mechanism, which involves the linear SiOO intermediate, the highly specific DCSs and rovibrational distributions obtained at low $E_{c}$ and $j$ values from QCT calculations (dominant backward scattering, vibrational inversion peaked at $v^{\prime}=3$, and low rotational excitation) cannot be properly reproduced by statistical approaches. By contrast, at large $E_{c}$ or $j$ values, the energy and angular momentum partitioning into the product modes proceeds in an almost statistical manner, consistently with the dominant insertion mechanism, thus allowing the use of SQM and PST approaches to get a proper description of product state properties. Note, however, that some slight nonstatistical features persist at large $E_{c}$ or $j$ values for both the DCSs and rovibrational distributions, which are due to a remaining contribution of the abstraction mechanism (about 25\% of the total reactivity).
Finally, additional calculations performed at a collision energy of $135 \mathrm{meV}$ and a rotational temperature of $50 \mathrm{~K}$ for $\mathrm{O}_{2}$ allowed a comparison between the theoretical $\mathrm{SiO}\left(v^{\prime}\right)$ vibrational distribution and that obtained from the experimental measurement of Yamashiro et al. ${ }^{7}$ The partial agreement between the QCT and experimental distributions points out that the dominant abstraction mechanism associated with the singlet channel of the title reaction may explain the inverted vibrational distribution observed in experiment. Furthermore, the discrepancies observed for particular vibrational states suggest a significant contribution to reactivity of the additional triplet channel of the reaction, not accounted for in the present work, and likely proceeding through a direct mechanism. Theoretical works on the triplet channel of the $\mathrm{Si}+\mathrm{O}_{2}$ reaction or experimental measurement of the $\mathrm{O}\left({ }^{3} P\right) / \mathrm{O}\left({ }^{1} D\right)$ branching ratio would be very valuable to determine the exact contribution of each channel to reactivity.

\section{ACKNOWLEDGMENTS}

This work has been funded by the MEC (Spain) under Grant No. FIS2007-62006 and under the MEC-CSIC Project Ref. No. 200650I134. The authors would like to acknowledge the use of the computer resources of the Barcelona Supercomputing Center and that of the IDRIS French computing center. We also wish to thank Professor $\mathrm{K}$. Honma for sharing with us the preliminary experimental results.

\section{APPENDIX: PHASE SPACE THEORY IN THE $j \neq 0$ CASE}

\section{Coordinates and parameters}

The reactant atom-diatom system is described by the following set of 12 canonical coordinates: ${ }^{52}$ The total angular momentum $J$, its space-fixed component $J_{z}$, the orbital angular momentum $l$, the diatom rotational angular momentum $j$, their respective conjugated angles $\alpha, \beta, \alpha_{l}$, and $\alpha_{j}$, the distance $R$ between the atom and the diatom c.m., the diatom bound distance $r$, and their respective conjugate momenta $P_{R}$ and $p_{r}$. Besides, $\mu$ and $m$ are, respectively, the reactant atomdiatom and diatom reduced masses, and $r_{e}$ is the diatom equilibrium distance. $E$ is defined as the total energy with respect to the reactant bottom channel, $E_{c}$ is the collision energy, and $k=\sqrt{2 \mu E_{c}} / \hbar$ is the reactant momentum. The two equivalent product atom-diatom channels are defined by an analogous set of primed coordinates and parameters: $J^{\prime}, J_{z}^{\prime}$, $l^{\prime}, j^{\prime}, \alpha^{\prime}, \beta^{\prime}, \alpha_{l}^{\prime}, \alpha_{j}^{\prime}, R^{\prime}, r^{\prime}, P_{R}^{\prime}, p_{r}^{\prime}, \mu^{\prime}, m^{\prime}$, and $r_{e}^{\prime}$. All angular momenta are expressed in $\hbar$ units in the following. In this work, the diatomic molecules are described as rigidrotor anharmonic oscillators, for which the rovibrational energy levels are approximated by

$$
E_{v j}=h c \omega_{e}\left(v+\frac{1}{2}\right)-h c \omega_{e} x_{e}\left(v+\frac{1}{2}\right)^{2}+\frac{\hbar^{2}}{2 m r_{e}^{2}} j(j+1) .
$$

All parameters entering Eq. (A1) have been taken from the $a b$ initio PES. ${ }^{1}$ The total energy $E^{\prime}$ with respect to the product channel bottom is defined as 


$$
E^{\prime}=E+\Delta E_{0}=E_{c}+E_{v j}+\Delta E_{0},
$$

where $E_{v j}$ is the reactant diatom rovibrational energy, $E_{c}$ the collision energy, and $\Delta E_{0}$ the reaction exoergicity excluding the reactant and product zero point energies. The mechanical parameters used in this work are $m_{\mathrm{Si}}=28$ a.m.u., $m_{\mathrm{O}}=16$ a.m.u., $\quad r_{e}^{\mathrm{O}_{2}}=1.215 \AA, \quad \omega_{e}^{\mathrm{O}_{2}}=1546 \mathrm{~cm}^{-1}$, $\omega_{e} x_{e}^{\mathrm{O}_{2}}=8.88 \mathrm{~cm}^{-1}, \quad C_{6}^{\mathrm{Si}-\mathrm{O}_{2}}=52.86 \mathrm{eV} / \AA^{6}, \quad r_{e}^{\mathrm{SiO}}=1.52 \AA$, $\omega_{e}^{\mathrm{SiO}}=1154 \mathrm{~cm}^{-1}, \omega_{e} x_{e}^{\mathrm{SiO}}=6.34 \mathrm{~cm}^{-1}, C_{6}^{\mathrm{O}-\mathrm{SiO}}=14.42 \mathrm{eV} / \AA^{6}$, and $\Delta E_{0}=0.93 \mathrm{eV}$.

\section{Differential cross section}

The state-resolved reaction cross section for a process of the type $A+B C(v, j) \rightarrow A B\left(v^{\prime}, j^{\prime}\right)+C$ is given by ${ }^{9}$

$$
\sigma_{v^{\prime} j^{\prime}, v j}\left(E_{c}\right)=\int_{0}^{b_{\max }} 2 \pi b \wp_{v^{\prime} j^{\prime}, v j}\left(b, E_{c}\right) d b,
$$

where $b$ is the impact parameter, $b_{\max }$ its maximum value leading to reaction, and the opacity function $\wp_{v^{\prime} j^{\prime}, v j}\left(b, E_{c}\right)$ is the state-resolved reaction probability in terms of $b$. Since the orbital angular momentum $l$ is related to $b$ by $l=k b$, Eq. (A3) can be written as

$$
\sigma_{v^{\prime} j^{\prime}, v j}\left(E_{c}\right)=\frac{2 \pi}{k^{2}} \int_{0}^{l_{\max }} P_{v^{\prime} j^{\prime}, v j}\left(l, E_{c}\right) l d l,
$$

where $l_{\max }=k b_{\max }$ and $P_{v^{\prime} j^{\prime}, v j}\left(l, E_{c}\right)=\wp_{v^{\prime} j^{\prime}, v j}\left(b, E_{c}\right)$ is the opacity function in terms of $l$. The total DCS is defined as

$$
\sigma_{v^{\prime} j^{\prime}, v j}\left(\theta, E_{c}\right)=\frac{d \sigma_{v^{\prime} j^{\prime}, v j}\left(E_{c}\right)}{d \omega},
$$

where $d \omega=2 \pi \sin \theta d \theta$ is the differential solid angle. Considering Eqs. (A4) and (A5), the DCS might be rewritten as

$$
\sigma_{v^{\prime} j^{\prime}, v j}\left(\theta, E_{c}\right)=\frac{1}{k^{2} \sin \theta} \int_{0}^{l_{\max }} \frac{d P_{v^{\prime} j^{\prime}, v j}\left(l, E_{c}\right)}{d \theta} l d l,
$$

which leads to Eq. (5) if we state that

$$
P_{v^{\prime} j^{\prime}, v j}\left(\theta, l, E_{c}\right)=\frac{d P_{v^{\prime} j^{\prime}, v j}\left(l, E_{c}\right)}{d \theta},
$$

where $P_{v^{\prime} j^{\prime}, v j}\left(\theta, l, E_{c}\right) d \theta$ is the state-resolved reaction probability associated with a c.m. scattering angle within the range of $[\theta, \theta+d \theta]$. The probability $P_{v^{\prime} j^{\prime}, v j}\left(l, E_{c}, \theta\right)$ reads as

$$
P_{v^{\prime} j^{\prime}, v j}\left(\theta, l, E_{c}\right)=p_{v j}^{\mathrm{cap}}\left(l, E_{c}\right) \int_{|l-j|}^{l+j} \rho(J \mid l, j) P_{v^{\prime} j^{\prime}}^{P}\left(\theta, E^{\prime}, J\right) d J,
$$

where $p_{v j}^{\text {cap }}\left(l, E_{c}\right)$ is the complex formation probability, $\rho(J \mid l, j)$ is the total angular momentum distribution consistent with $l$ and $j$, and $P_{v^{\prime} j^{\prime}}^{P}\left(\theta, E^{\prime}, J\right)$ is the density of probability that the intermediate complex dissociates toward the $\left(v^{\prime}, j^{\prime}\right)$ state, at c.m. scattering angle $\theta$, consistent with the total energy $E^{\prime}$ and total angular momentum $J$. Since we consider an isotropic interaction potential between the fragments, $p_{v j}^{\text {cap }}\left(l, E_{c}\right)$ and $P_{v^{\prime} j^{\prime}}^{P}\left(\theta, E^{\prime}, J\right)$ can be computed within the framework of PST, as detailed in the next subsections. The DCS formulation via Eqs. (A5)-(A8) extends the one of

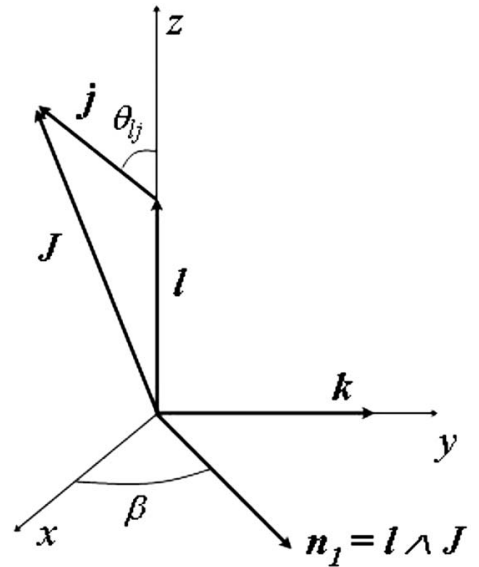

FIG. 9. Scheme representing the link between the $\boldsymbol{J}, \boldsymbol{l}, \boldsymbol{j}$ vectors and their related angles.

Ref. 13, in which the treatment was limited to a rotationaly frozen reactant diatom $(j=0)$. Notice that, in the present $j \neq 0$ case, the c.m. scattering angle explicitly depends on the orientation of $\boldsymbol{J}$.

\section{Complex formation probability}

Since the process of interest is assumed to be governed by long-range isotropic forces, the intermediate complex formation probability $p_{v j}^{\text {cap }}\left(l, E_{c}\right)$ is estimated via a classical capture model, ${ }^{42}$

$$
p_{v j}^{\operatorname{cap}}\left(l, E_{c}\right)= \begin{cases}1 & \text { if } l \leqslant l_{\max } \\ 0 & \text { if } l>l_{\max }\end{cases}
$$

where $l_{\max }$ is the maximal value of $l$ consistent with complex formation and is obtained by equating the height of the entrance centrifugal barrier to the collision energy $E_{c} \cdot{ }^{37}$

\section{Total angular momentum distribution}

We consider a space-fixed orthonormal coordinate system $\{\boldsymbol{O}, \boldsymbol{x}, \boldsymbol{y}, \boldsymbol{z}\}$ and assume that $(i)$ the reactant relative momentum $\boldsymbol{k}$ lies along the $y$ axis and (ii) the initial orbital angular momentum $\mathbf{I}$ lies along $z$ (see Fig. 9). The $J_{z}$ conjugate angle, $\beta$, locates the $n_{1}=l \wedge J$ line of nodes with respect to the $x$ axis. Since $\boldsymbol{j}$ is randomly oriented, the distribution of $J$ for a given value of $l, \rho(J \mid l, j)$, is given by

$$
\rho(J \mid l, j)=\frac{1}{2} \int_{0}^{\pi} \delta\left(J-\left\{j^{2}+l^{2}+2 l j \cos \theta_{l j}\right\}^{1 / 2}\right) \sin \theta_{l j} d \theta_{l j},
$$

where $\cos \theta_{l j}=\boldsymbol{j} \cdot \boldsymbol{l} / l j$ and $\delta$ is the Dirac function. The latter integral can be analytically evaluated, or numerically, by using a Monte Carlo method ${ }^{53}$ and assuming that

$$
\begin{aligned}
\delta(J- & \left.\left\{j^{2}+l^{2}+2 l j \cos \theta_{l j}\right\}\right) \\
\approx & \Theta\left[(J+1 / 2)-\left(j^{2}+l^{2}+2 l j \cos \theta_{l j}\right)\right] \\
& \times \Theta\left[\left(j^{2}+l^{2}+2 l j \cos \theta_{l j}\right)-(J-1 / 2)\right],
\end{aligned}
$$

where $\Theta$ is the Heaviside function. 


\section{Complex decay probability}

Within the framework of PST, the complex decay probability $P_{v^{\prime} j^{\prime}}^{P}\left(\theta, E^{\prime}, J\right)$ writes as

$$
P_{v^{\prime} j^{\prime}}^{P}\left(\theta, E^{\prime}, J\right)=\frac{2 d N_{v^{\prime} j^{\prime}}^{P}\left(\theta, E^{\prime}, J\right) / d \theta}{2 N^{P}\left(E^{\prime}, J\right)+N^{R}(E, J)},
$$

where $d N_{v^{\prime} j^{\prime}}^{P}\left(\theta, E^{\prime}, J\right) / d \theta$ is the density of product states consistent with $v^{\prime}, j^{\prime}, \theta, E^{\prime}$, and $J$, and $N^{R}(E, J)$ and $N^{P}\left(E^{\prime}, J\right)$ are the total numbers of reactant and product states, respectively, energetically accessible and consistent with the conservation of the total angular momentum $J$. A factor of 2 appears in Eq. (A12) due to the degeneracy of the product channel. The total number of product states $N^{P}\left(E^{\prime}, J\right)$ is given by

$$
N^{P}\left(E^{\prime}, J\right)=\sum_{v^{\prime}=0}^{v_{\max }^{\prime}} N_{v^{\prime}}^{\mathrm{rot}}\left(E^{\prime}, J\right)
$$

where $v_{\max }^{\prime}$ is the highest vibrational quantum number consistent with $E^{\prime}$ and $N_{v^{\prime}}^{\mathrm{rot}}\left(E^{\prime}, J\right)$ is the number of orbital and rotational states consistent with a diatom rotational energy lower than $E^{\prime}-E_{v^{\prime}}^{\prime}$. The value of $N_{v^{\prime}}^{\text {rot }}\left(E^{\prime}, J\right)$ is estimated via

$$
\begin{aligned}
N_{v^{\prime}}^{\mathrm{rot}}\left(E^{\prime}, J\right)= & \int \frac{d \Gamma^{\prime}}{(2 \pi)^{4}} \delta\left(J^{\prime}-J\right) \\
& \times \Theta\left(j_{\max }^{\prime}-j^{\prime}\right) \Theta\left(l_{\max }^{\prime}-l^{\prime}\right) \\
& \times \Theta\left(J-\left|l^{\prime}-j^{\prime}\right|\right) \Theta\left(l^{\prime}+j^{\prime}-J\right),
\end{aligned}
$$

where the $\delta$ and $\Theta$ functions are the Dirac and Heaviside functions, respectively. The $\delta$ function ensures that $J^{\prime}=J$, while the $\Theta$ functions restrict the integration domain over $j^{\prime}$ and $l^{\prime}$ and impose the triangular inequality resulting from $\boldsymbol{J}=\boldsymbol{j}+\boldsymbol{l}$. The differential phase space volume associated with rotational and orbital degrees of freedom is given by $d \Gamma^{\prime}$ $=d J^{\prime} d J_{z}^{\prime} d l^{\prime} d j^{\prime} d \alpha^{\prime} d \beta^{\prime} d \alpha_{l}^{\prime} d \alpha_{j}^{\prime}$. The values $j_{\max }^{\prime}$ and $l_{\max }^{\prime}$ are the maximal available rotational action and orbital angular momenta, respectively, consistent with the $v^{\prime}$ value. The integral of Eq. (A14) can be evaluated by a Monte Carlo numerical method ${ }^{53}$ after analytical integration over angles, $J^{\prime}$ and $J_{z}^{\prime} \cdot{ }^{13}$ The total number of reactant states $N^{R}(E, J)$ can be computed in the same way as $N^{P}\left(E^{\prime}, J\right)$ by using the coordinates and parameters of the reactant channel.

The density of product states $d N_{v^{\prime} j^{\prime}}^{P}\left(\theta, E^{\prime}, J\right) / d \theta$ can be evaluated in a similar way as $N_{v^{\prime}}^{\text {rot }}\left(E^{\prime}, J\right)$, adding extra constraints which specify the c.m. scattering angle as well as the product diatom rotational action into the integral of Eq. (A14). Nevertheless, as it will be shown in the next subsection, the definition of $\theta$ depends on the $\mathbf{J}$ orientation described by the $\alpha$ and $\beta$ angles (see Fig. 10). Consequently, the density of product states consistent with $\theta$ and $\mathbf{J}$ is given by

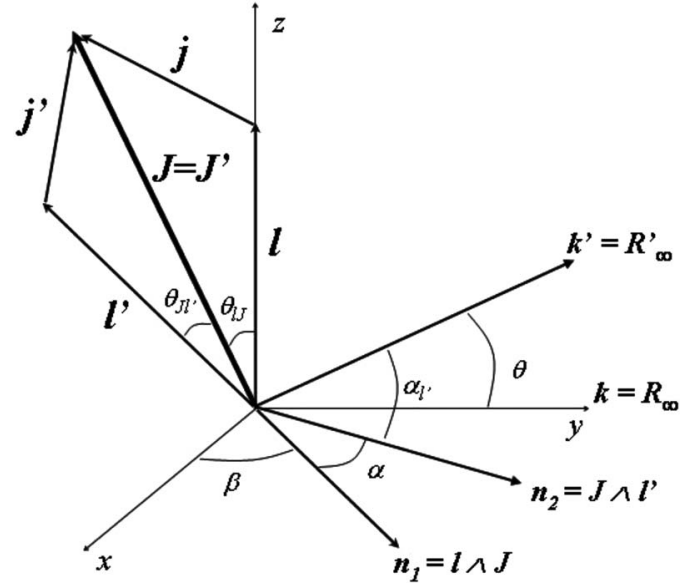

FIG. 10. Scheme representing the vectors and angles needed to derive the expression of $\theta_{\text {scat }}$.

$$
\begin{aligned}
\frac{d N_{v^{\prime} j^{\prime}}^{P}\left(\theta, E^{\prime}, J, \alpha, \beta\right)}{d \theta}= & \int \frac{d \Gamma^{\prime \prime}}{(2 \pi)^{4}} \delta\left(J^{\prime}-J\right) \\
& \times \Theta\left(j_{\max }^{\prime}-j^{\prime \prime}\right) \Theta\left(l_{\max }^{\prime}-l^{\prime}\right) \\
& \times \Theta\left(J-\left|l^{\prime}-j^{\prime \prime}\right|\right) \Theta\left(l^{\prime}+j^{\prime \prime}-J\right) \\
& \times \delta\left(j^{\prime \prime}-j^{\prime}\right) \delta\left[\theta-f\left(\Gamma^{\prime}, \alpha, \beta\right)\right],
\end{aligned}
$$

where $\quad d \Gamma^{\prime \prime}=d J^{\prime} d J_{z}^{\prime} d l^{\prime} d j^{\prime \prime} d \alpha^{\prime} d \beta^{\prime} d \alpha_{l}^{\prime} d \alpha_{j}^{\prime}$. The relation $f\left(\Gamma^{\prime}, \alpha, \beta\right)$ linking $\theta$ to the reactant and product phase space variables is derived in the next section [Eqs. (A20)-(A28)]. The density of product states $d N_{v^{\prime} j^{\prime}}^{P}\left(\theta, E^{\prime}, J\right) / d \theta$ is then computed as the average of the previous density over the $\alpha$ and $\beta$ angles,

$$
\frac{d N_{v^{\prime} j^{\prime}}^{P}\left(\theta, E^{\prime}, J\right)}{d \theta}=\frac{1}{(2 \pi)^{2}} \int d \alpha d \beta \frac{d N_{v^{\prime} j^{\prime}}^{P}\left(\theta, E^{\prime}, J, \alpha, \beta\right)}{d \theta} .
$$

As stated previously, within the framework of complexforming atom-diatom reactions, the DCS must be computed by using Eq. (6) to approximately account for parity conservation into a classical approach. ${ }^{14,15}$ As a result, it is necessary to estimate via Eq. (7) the zero-helicity state-resolved DCS, $\sigma_{v^{\prime} j^{\prime}\left(\Omega^{\prime}=0\right), v j(\Omega=0)}\left(\theta, E_{c}\right), \Omega$ being the projection of $\boldsymbol{j}$ (or equivalently $\boldsymbol{J}$ ) on $\boldsymbol{k}$ (see Fig. 9). The density of probability $P_{v^{\prime} j^{\prime}\left(\Omega^{\prime}=0\right), v j(\Omega=0)}\left(\theta, E_{c}\right)$ entering this equation is defined as

$$
\begin{aligned}
& P_{v^{\prime} j^{\prime}\left(\Omega^{\prime}=0\right), v j(\Omega=0)}\left(\theta, l, E_{c}\right) \\
& \quad=p_{v j, \Omega=0}^{\operatorname{cap}}\left(l, E_{c}\right) \int_{|l-j|}^{l+j} \rho(J \mid l, j, \Omega=0) P_{v^{\prime} j^{\prime}, \Omega^{\prime}=0}^{P}\left(\theta, E^{\prime}, J\right) d J,
\end{aligned}
$$

where $p_{v j, \Omega=0}^{\text {cap }}\left(l, E_{c}\right)$ is evaluated as previously through Eq. (A9). Besides, $\Omega=0$ only if $\boldsymbol{j}$ and $\boldsymbol{l}$ are aligned. Consequently, the distribution of total angular momentum $\rho(J \mid l, j, \Omega=0)$ is given by 


$$
\rho(J \mid l, j, \Omega=0)=\frac{1}{2}[\delta(J-|l-j|)+\delta(J-|l+j|)] .
$$

The complex decay probability $P_{v^{\prime} j^{\prime}, \Omega^{\prime}=0}^{P}\left(\theta, E^{\prime}, J\right)$ of Eq. (A17) is evaluated in the same way as previously, i.e., by replacing $d N_{v^{\prime} j^{\prime}}^{P}\left(\theta, E^{\prime}, J\right) / d \theta$ by $d N_{v^{\prime} j^{\prime}, \Omega^{\prime}=0}^{P}\left(\theta, E^{\prime}, J\right) / d \theta$ in Eq. (A12), where the zero-helicity state-resolved density of product states is computed as follows:

$$
\begin{aligned}
\frac{d N_{v^{\prime} j^{\prime}, \Omega^{\prime}=0}^{P}\left(\theta, E^{\prime}, J\right)}{d \theta}= & \frac{1}{(2 \pi)^{2}} \int \frac{d \Gamma^{\prime \prime}}{(2 \pi)^{4}} d \alpha d \beta \delta\left(J^{\prime}-J\right) \\
& \times \Theta\left(j_{\max }^{\prime}-j^{\prime \prime}\right) \Theta\left(l_{\max }^{\prime}-l^{\prime}\right) \\
& \times \Theta\left(J-\left|l^{\prime}-j^{\prime \prime}\right|\right) \Theta\left(l^{\prime}+j^{\prime \prime}-J\right) \\
& \times \delta\left(j^{\prime \prime}-j^{\prime}\right) \delta\left[\theta-f\left(\Gamma^{\prime}, \alpha, \beta\right)\right] \delta\left(\Omega^{\prime}\right) .
\end{aligned}
$$

The above equation is equivalent to Eq. (A16) with an additional constraint on $\Omega^{\prime}$. The integral can be numerically evaluated, knowing the link between $\Omega^{\prime}$ and the product canonical coordinate which is described below.

\section{Center-of-mass scattering angle}

The link between $\theta$, the c.m. scattering angle between $\boldsymbol{k}$ and $\boldsymbol{k}^{\prime}$, and the coordinates describing the system are illustrated in Fig. 10. Let $\{\boldsymbol{O}, \boldsymbol{x}, \boldsymbol{y}, \boldsymbol{z}\}$ be a space-fixed orthonormal frame and assume that $\boldsymbol{l}$ and $\boldsymbol{k}$ lie along the $z$ and $y$ axis, respectively. The $\boldsymbol{k}^{\prime}$ vector, which is asymptotically parallel to $\boldsymbol{R}^{\prime} \equiv \boldsymbol{R}_{\infty}^{\prime}$ in the product channel, is located by five rotations with respect to the $x$ axis. The five rotations, labeled $R_{i}(i=1-5)$, are described below.

$R_{1}$ is the rotation by the $\beta$ angle about the $z$ axis which locates the direction of the $n_{1}=l \wedge \boldsymbol{J}$ line of nodes. The rotation matrix is given by

$$
\mathbf{R}_{1}=\left(\begin{array}{ccc}
\cos \beta & -\sin \beta & 0 \\
\sin \beta & \cos \beta & 0 \\
0 & 0 & 1
\end{array}\right) .
$$

$R_{2}$ is the rotation by the angle $\theta_{l J}$ about $\boldsymbol{n}_{1}$, which locates the direction of $J$ with respect to the $z$ axis. The rotation matrix reads

$$
\mathbf{R}_{2}=\left(\begin{array}{ccc}
1 & 0 & 0 \\
0 & \cos \theta_{l J} & -\sin \theta_{l J} \\
0 & \sin \theta_{l J} & \cos \theta_{l J}
\end{array}\right)
$$

Since $\boldsymbol{j}=\boldsymbol{J}-\boldsymbol{l}, \cos \theta_{l J}$ is given by

$$
\cos \theta_{l J}=\frac{J^{2}+l^{2}-j^{2}}{2 l J} .
$$

$R_{3}$ is the rotation by the angle $\alpha$ at about $\boldsymbol{J}$ which locates the direction of the $\boldsymbol{n}_{2}=\boldsymbol{J} \wedge l^{\prime}$ line of nodes with respect to $\boldsymbol{n}_{1}$. The rotation matrix is

$$
\mathbf{R}_{3}=\left(\begin{array}{ccc}
\cos \alpha & -\sin \alpha & 0 \\
\sin \alpha & \cos \alpha & 0 \\
0 & 0 & 1
\end{array}\right) .
$$

$R_{4}$ is the rotation by the angle $\theta_{J l^{\prime}}$ about $\boldsymbol{n}_{2}$ which locates the direction of $\boldsymbol{l}^{\prime}$ with respect to $\boldsymbol{J}$. The rotation matrix is

$$
\mathbf{R}_{4}=\left(\begin{array}{ccc}
1 & 0 & 0 \\
0 & \cos \theta_{J l^{\prime}} & -\sin \theta_{J l^{\prime}} \\
0 & \sin \theta_{J l^{\prime}} & \cos \theta_{J l^{\prime}}
\end{array}\right) .
$$

As $\boldsymbol{j}^{\prime}=\boldsymbol{J}-\boldsymbol{l}^{\prime}, \cos \theta_{J l^{\prime}}$ is then

$$
\cos \theta_{J l^{\prime}}=\frac{J^{2}+l^{\prime 2}-j^{\prime 2}}{2 J l^{\prime}} .
$$

$R_{5}$ is the rotation by the angle $\alpha_{l^{\prime}}$ about $\boldsymbol{l}^{\prime}$ which locates the direction of $\boldsymbol{k}^{\prime}$ with respect to the $\boldsymbol{n}_{2}$. The rotation matrix is

$$
\mathbf{R}_{5}=\left(\begin{array}{ccc}
\cos \alpha_{l^{\prime}} & -\sin \alpha_{l^{\prime}} & 0 \\
\sin \alpha_{l^{\prime}} & \cos \alpha_{l^{\prime}} & 0 \\
0 & 0 & 1
\end{array}\right) .
$$

Within the $\{\boldsymbol{O}, \boldsymbol{x}, \boldsymbol{y}, \boldsymbol{z}\}$ frame, the $\boldsymbol{u}_{k^{\prime}}$ unit vector along $\boldsymbol{k}^{\prime}$ is given by

$$
\boldsymbol{u}_{k^{\prime}}=\mathbf{R}_{5} \mathbf{R}_{4} \mathbf{R}_{3} \mathbf{R}_{2} \mathbf{R}_{1}\left(\begin{array}{l}
0 \\
1 \\
0
\end{array}\right) .
$$

The c.m. scattering angle is, thus, given by

$$
\begin{aligned}
\theta= & f\left(\Gamma^{\prime}, \alpha, \beta\right)=\arccos \left(\boldsymbol{u}_{k^{\prime}} \cdot \boldsymbol{y}\right) \\
= & \arccos \left\{\sin \beta\left(\cos \alpha \cos \alpha_{l^{\prime}}-\sin \alpha \sin \alpha_{l^{\prime}} \cos \theta_{J l^{\prime}}\right)\right. \\
& +\cos \beta\left[\cos \theta_{l J}\left(\sin \alpha \cos \alpha_{l^{\prime}}+\cos \alpha \sin \alpha_{l^{\prime}} \cos \theta_{J l^{\prime}}\right)\right. \\
& \left.\left.-\sin \theta_{l J}\left(\sin \alpha \cos \alpha_{l^{\prime}}+\cos \alpha \sin \alpha_{l^{\prime}} \cos \theta_{J l^{\prime}}\right)\right]\right\} .
\end{aligned}
$$

The above relation is used in the numerical Monte Carlo computation of Eqs. (A15) and (A19), where the Dirac function is approximated by the product of two Heaviside functions in an analogous way as Eq. (A11).

\section{Helicity angular momentum}

Figure 11 shows some relevant vectors and angles of the problem. The three vectors $\boldsymbol{l}^{\prime}, \boldsymbol{x}$, and $\boldsymbol{y}$ define an orthogonal frame. $\boldsymbol{j}^{\prime}$ is contained in the plane defined by $\boldsymbol{l}^{\prime}$ and $\boldsymbol{y}$. Its projection on the axis carrying $\boldsymbol{y}$ is called $j_{y}^{\prime} . j_{y}^{\prime}$ and is related to $j^{\prime}$ by

$$
j_{y}^{\prime}=\sin \theta_{l^{\prime} j^{\prime} j^{\prime}} .
$$

Squaring the equation

$$
\boldsymbol{J}=\boldsymbol{j}^{\prime}+\boldsymbol{l}^{\prime}
$$

leads immediately to

$$
\cos \theta_{l^{\prime} j^{\prime}}=\frac{J^{2}-j_{2}^{\prime}-l^{\prime 2}}{2 j^{\prime} l^{\prime}} .
$$

From Eq. (A29), we thus have 


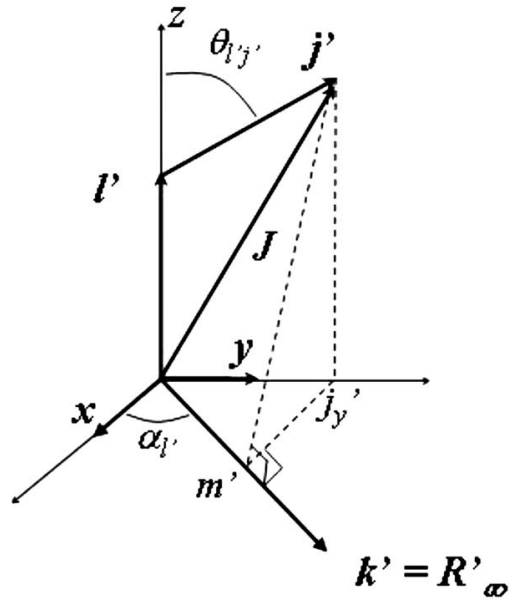

FIG. 11. Scheme representing the vectors and angles needed to derive the expression of $\Omega^{\prime}$.

$$
j_{y}^{\prime}=\left[1-\left(\frac{J^{2}-j^{\prime 2}-l^{\prime 2}}{2 j^{\prime} l^{\prime}}\right)^{2}\right]^{1 / 2} j^{\prime} .
$$

Notice that the projections of $\boldsymbol{j}^{\prime}$ and $\boldsymbol{j}_{\boldsymbol{y}}^{\prime}$ on the $R_{\infty}^{\prime}$ axis are both equal to $\boldsymbol{\Omega}^{\prime}$. Since the angle between $\boldsymbol{R}_{\infty}^{\prime}$ and $\boldsymbol{j}_{\boldsymbol{y}}^{\prime}$ is equal to $\pi / 2-\alpha_{l^{\prime}}$, we finally obtain

$$
\Omega^{\prime}=\sin \alpha_{l}^{\prime}\left[1-\left(\frac{J^{2}-j^{\prime 2}-l^{\prime 2}}{2 j^{\prime} l^{\prime}}\right)^{2}\right]^{1 / 2} j^{\prime} .
$$

The above relation is used in the numerical Monte Carlo computation of Eq. (A19), where the Dirac function is approximated by the product of two Heaviside functions in an analogous way as Eq. (A11).

${ }^{1}$ F. Dayou and A. Spielfiedel, J. Chem. Phys. 119, 4237 (2003).

${ }^{2}$ S. D. Le Picard, A. Canosa, G. Pineau des Forêts, C. Rebrion-Rowe, and B. R. Rowe, Astron. Astrophys. 372, 1064 (2001).

${ }^{3}$ S. D. Le Picard, A. Canosa, D. Reignier, and T. Stoecklin, Phys. Chem. Chem. Phys. 4, 3659 (2002).

${ }^{4}$ I. Adamovic and M. Gordon, J. Phys. Chem. A 108, 8395 (2004).

${ }^{5}$ M. H. P. M. Agrawal, L. M. Raff, and R. Komanduri, J. Chem. Phys. 124, 134306 (2006)

${ }^{6}$ W. Lu, C. Wang, V. Nguyen, M. Schmidt, M. Gordon, and K. Ho, J. Phys. Chem. A 107, 6936 (2003).

${ }^{7}$ R. Yamashiro, Y. Matsumoto, and K. Honma, J. Chem. Phys. 128, 084308 (2008).

${ }^{8}$ F. Dayou, W.-Ü. L. Tchang-Brillet, and M. Monnerville, J. Chem. Phys. 123, 4306 (2005)

${ }^{9}$ R. D. Levine and R. B. Bernstein, Molecular Reaction Dynamics and Chemical Reactivity (Oxford University Press, New York, 1987).

${ }^{10}$ F. Dayou, Ph.D. thesis, Université P. et M. Curie (ParisVI), (2002).

${ }^{11}$ E. J. Rackham, F. Huarte-Larrañaga, and D. E. Manolopoulos, Chem. Phys. Lett. 343, 356 (2001).

${ }^{12}$ E. J. Rackham, T. Gonzalez-Lezana, and D. E. Manolopoulos, J. Chem. Phys. 119, 12895 (2003)

${ }^{13}$ P. Larrégaray, L. Bonnet, and J.-C. Rayez, J. Phys. Chem. A 110, 1552 (2006).
${ }^{14}$ L. Bonnet, P. Larrégaray, J. C. Rayez, and T. González-Lezana, Phys. Chem. Chem. Phys. 8, 3951 (2006).

${ }^{15}$ L. Bonnet, P. Larregaray, and J. C. Rayez, Phys. Chem. Chem. Phys. 9, 3228 (2007).

${ }^{16}$ F. J. Aoiz, V. Saéz-Rábanos, T. González-Lezana, and D. E. Manolopoulos, J. Chem. Phys. 126, 161101 (2007).

${ }^{17}$ T. González-Lezana, O. Roncero, P. Honvault, J.-M. Launay, N. Bulut, F. Javier Aoiz, and L. Bañares, J. Chem. Phys. 125, 94314 (2006).

${ }^{18}$ N. Balucani, G. Capozza, E. Segoloni, A. Russo, R. Bobbenkamp, P. Casavecchia, T. Gonzalez-Lezana, E. J. Rackham, L. Bañares, and F. J. Aoiz, J. Chem. Phys. 122, 4309 (2005).

${ }^{19}$ L. Bañares, F. J. Aoiz, T. González-Lezana, V. J. Herrero, and I. Tanarro, J. Chem. Phys. 123, 224301 (2005).

${ }^{20}$ N. Balucani, P. Casavecchia, L. Bañares, F. J. Aoiz, T. González-Lezana, P. Honvault, and J.-M. Launay, J. Phys. Chem. A 110, 817 (2006).

${ }^{21}$ S. Y. Lin and H. Guo, J. Phys. Chem. A 110, 5305 (2006).

${ }^{22}$ A. L. Van Wyngarden, K. A. Marr, K. A. Boering, J. J. Lin, Y. T. Lee, S.-Y. Lin, H. Guo, and G. Lendvay, J. Am. Chem. Soc. 129, 2866 (2007).

${ }^{23}$ P. Bargueño, T. González-Lezana, P. Larrégaray, L. Bonnet, and J. C. Rayez, Phys. Chem. Chem. Phys. 9, 1127 (2007).

${ }^{24}$ K. Kleinermanns and R. Schinke, J. Chem. Phys. 80, 1440 (1984).

${ }^{25}$ A. J. H. Meijer and E. M. Goldfield, J. Chem. Phys. 108, 5404 (1998).

${ }^{26}$ P. Halvick and J. C. Rayez, Chem. Phys. 131, 375 (1989).

${ }^{27}$ D. G. Truhlar and J. T. Muckerman, Atom-Molecule Collision Theory. A guide for the Experimentalists, edited by R. B. Bernstein (Plenum, New York, 1979), Chap. 16.

${ }^{28}$ L. Bonnet and J. C. Rayez, Chem. Phys. Lett. 277, 183 (1997).

${ }^{29}$ L. Bañares, F. J. Aoiz, P. Honvault, and J.-M. Launay, J. Phys. Chem. A 108, 1616 (2004).

${ }^{30}$ F. J. Aoiz, V. Sáez-Rábanos, B. Martínez-Haya, and T. González-Lezana, J. Chem. Phys. 123, 4101 (2005).

${ }^{31}$ R. T. Pack, J. Chem. Phys. 60, 633 (1974).

${ }^{32}$ P. McGuire and D. J. Kouri, J. Chem. Phys. 60, 2488 (1974).

${ }^{33}$ K. McLenithan and D. Secrest, J. Chem. Phys. 80, 2480 (1984).

${ }^{34}$ B. R. Johnson, J. Comput. Phys. 13, 445 (1973).

${ }^{35}$ R. N. Zare, Angular Momentum: Understanding Spatial Aspects in Chemistry and Physics (Wiley-Interscience, New York, 1988).

${ }^{36}$ J. C. Light, J. Chem. Phys. 40, 3221 (1964).

${ }^{37}$ P. Pechukas and J. C. Light, J. Chem. Phys. 42, 3281 (1965).

${ }^{38}$ J. C. Light, Discuss. Faraday Soc. 44, 14 (1967).

${ }^{39}$ J. C. Keck, J. Chem. Phys. 29, 410 (1958).

${ }^{40}$ E. E. Nikitin, Theory of Elementary Atomic and Molecular Processes in Gases (Clarendon, Oxford, 1974).

${ }^{41}$ D. G. Truhlar, B. C. Garret, and S. J. Klippenstein, J. Phys. Chem. 100, 12771 (1996).

${ }^{42}$ P. Langevin, Ann. Chim. Phys. 5, 245 (1905).

${ }^{43}$ T. González-Lezana, Int. Rev. Phys. Chem. 26, 29 (2007).

${ }^{44}$ L. Bonnet and J. C. Rayez, Eur. Phys. J. D 38, 65 (2006).

${ }^{45}$ J. O. Hirschfelder, C. F. Curtiss, and R. B. Bird, Molecular Theory of Gases and Liquids (Wiley, New York, 1954).

${ }^{46}$ P. Larrégaray, L. Bonnet, and J.-C. Rayez, J. Chem. Phys. 127, 084308 (2007).

${ }^{47}$ F. J. Aoiz, V. Sáez-Rábanos, and T. González-Lezana (to be published).

${ }^{48}$ E. Carmona-Novillo, T. González-Lezana, O. Roncero, P. Honvault, J.-M. Launay, N. Bulut, F. Javier Aoiz, L. Bañares, A. Trottier, and E. Wrede, J. Chem. Phys. 128, 014304 (2008).

${ }^{49}$ L. Bonnet, J. Chem. Phys. 128, 044109 (2008).

${ }^{50}$ D. Case and D. Herschbach, J. Chem. Phys. 64, 4212 (1976).

${ }^{51}$ H. Akagi, Y. Fujimura, and O. Kajimoto, J. Chem. Phys. 110, 7264 (1999).

${ }^{52}$ D. M. Wardlaw and R. A. Marcus, J. Chem. Phys. 83, 3462 (1985).

${ }^{53}$ J. P. Nougier, Methodes de Calcul Numérique (Masson, Paris, 1983). 